Supporting Information for

\title{
Metal-free Mediated Meerwein-type Reaction: A Radical Cascade Arylation/Aryl Migration/Desulfonylation of Conjugated Alkenes
}

\author{
Zhangqin Ni, Xin Huang, Yuanjiang Pan * \\ Department of Chemistry, Zhejiang University, Hangzhou, 310027, China
}

\section{Content}

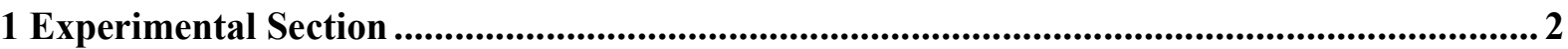

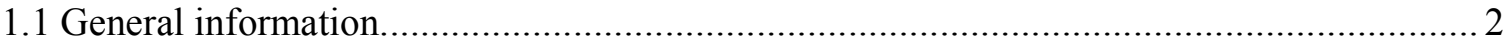

1.2 General procedure for the preparation of $N$-phenyl- $N$-(phenylsulfonyl)methacrylamide $\ldots . . .2$

2 Experimental Procedures and Spectral Data .................................................................2

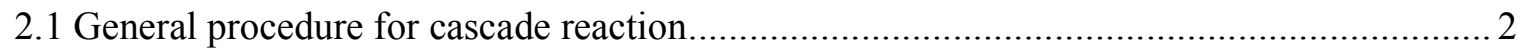

2.2 Substrate scope of $\alpha$-all-carbon quaternary stereocenters amides.................................. 3

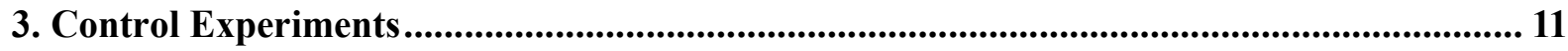

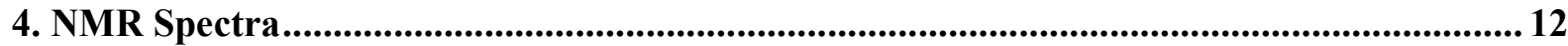




\section{Experimental Section}

\subsection{General information}

All chemicals were obtained from commercial suppliers and used without further purification. All reactions were conducted in oven-dried schlenk tubes under nitrogen atmosphere. Some ${ }^{1} \mathrm{H}$-NMR and ${ }^{13} \mathrm{C}-\mathrm{NMR}$ spectra were recorded on Bruker Avance 400 spectrometer at $400 \mathrm{MHz}$ in $d_{6}$-DMSO. The Others ${ }^{1} \mathrm{H}$ NMR and ${ }^{13} \mathrm{C}$ NMR spectra were recorded on Bruker AVANCE DMX-500 spectrometry at 500MHz in $d_{6}$-DMSO. Data for ${ }^{1} \mathrm{H}$-NMR are reported as follows: chemical shift $(\delta \mathrm{ppm})$, multiplicity, coupling constant $(\mathrm{Hz})$, and integration. Data for ${ }^{13} \mathrm{C}-\mathrm{NMR}$ are reported in terms of chemical shift ( $\delta \mathrm{ppm})$. Melting point is recorded on X4 micro melting-point apparatus. IR spectra are recorded on Bruker ALPHA FT-IR spectrometer. Flash column chromatographic purification of products was accomplished using forced-flow chromatography on neutral aluminum oxide (200-300 mesh).

\subsection{General procedure for the preparation of $N$-phenyl- $N$-(phenylsulfonyl)methacrylamide}<smiles>[R][R]1=C=CC=C(S(=O)(=O)Nc2[R]#cccc2)C1</smiles>

In a dry $100 \mathrm{~mL}$ round-bottom flask, a mixture of aniline $(10 \mathrm{mmol})$ in $20 \mathrm{~mL}$ of pyridine were stirred at room temperature. The benzene sulfochloride (11 mmol, 1.1 equiv) in $20 \mathrm{~mL}$ of pyridine was added slowly by a dropping funnel. The reaction was monitored by TLC. After the reaction completed, the mixture was evaporated under reduced pressure and extracted by ethyl acetate. The product was purified by silica gel column chromatography.

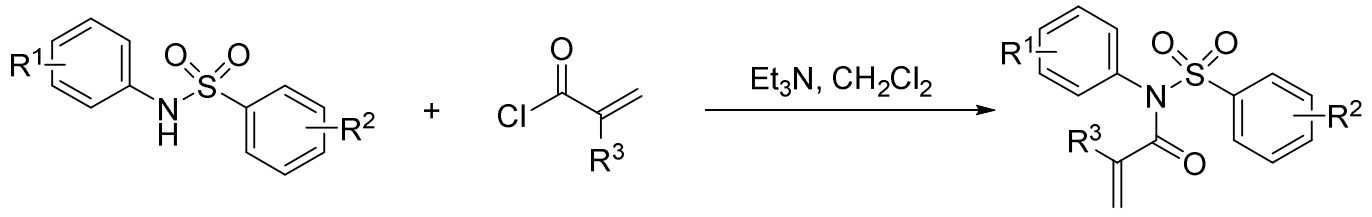

In a dry $100 \mathrm{~mL}$ round-bottom flask, a mixture of $N$-phenylbenzenesulfonamide (10 mmol) and $\mathrm{Et}_{3} \mathrm{~N}(30 \mathrm{mmol})$ in $20 \mathrm{~mL}$ of $\mathrm{CH}_{2} \mathrm{Cl}_{2}$ were stirred in ice bath. The acryloyl chloride (12 mmol, 1.2 equiv) in $10 \mathrm{~mL}$ of $\mathrm{CH}_{2} \mathrm{Cl}_{2}$ was added slowly by a dropping funnel. The reaction was monitored by TLC. After the reaction completed, the mixture was evaporated under reduced pressure and extracted by ethyl acetate. The product was purified by silica gel column chromatography.

\section{Experimental Procedures and Spectral Data}

\subsection{General procedure for cascade reaction}<smiles>C=C(C)C(=O)N(c1ccccc1)S(=O)(=O)c1ccccc1</smiles>

In a dry $25 \mathrm{~mL}$ oven-dried schlenk tubes, $N$-phenyl- $N$-(phenylsulfonyl)methacrylamide $(0.5 \mathrm{mmol})$, aniline $(1.5$ mmol, 3.0 equiv), TBAI ( $0.05 \mathrm{mmol}, 0.1$ equiv), phenanthroline ( $0.5 \mathrm{mmol}, 1$ equiv) were dissolved in the mixture of $1 \mathrm{~mL} \mathrm{MeNO}{ }_{2}$ and $50 \mu \mathrm{L} \mathrm{H}_{2} \mathrm{O}$ under an atmosphere of dry nitrogen at room temperature. Tert-butyl nitrite (1.75 mmol, 3.5 equiv) was then added with a syringe. The reaction mixture was heated to $110{ }^{\circ} \mathrm{C}$ for $10 \mathrm{~h}$. Then the solution was cooled to r.t., washed with a saturated aqueous solution of $\mathrm{NaCl}$, extracted by ethyl acetate and dried over by $\mathrm{Na}_{2} \mathrm{SO}_{4}$. The product was purified by neutral aluminum oxide column chromatography. 


\subsection{Substrate scope of $\alpha$-all-carbon quaternary stereocenters amides}<smiles>CC(Cc1ccc(Cl)cc1)(C(=O)Nc1ccccc1)c1ccccc1</smiles>

3-(4-chlorophenyl)-2-methyl- $N, 2$-diphenylpropanamide (3a)

Isolated with $96 \mathrm{mg}(55 \%)$, Yellow solid, m.p. $130-131^{\circ} \mathrm{C}$

IR 3410, 3345,3055,1677,1600,1535,1494,1439,1310,1242,1093,1014,758,671

${ }^{1}$ H NMR $\left(d_{6}\right.$-DMSO, $\left.400 \mathrm{MHz}\right) \delta: 9.20(\mathrm{~s}, 1 \mathrm{H}), 7.57(\mathrm{~m}, 2 \mathrm{H}), 7.34(\mathrm{~m}, 4 \mathrm{H}), 7.28(\mathrm{~m}, 3 \mathrm{H}), 7.21(\mathrm{~d}, J=8.4 \mathrm{~Hz}, 2 \mathrm{H})$, 7.04 (t, $J=7.4 \mathrm{~Hz}, 1 \mathrm{H}), 6.95$ (d, $J=8.4 \mathrm{~Hz}, 2 \mathrm{H}), 3.48$ (d, $J=13.2 \mathrm{~Hz} ., 1 \mathrm{H}), 3.17$ (d, $J=13.2 \mathrm{~Hz}, 1 \mathrm{H}), 1.48$ (s, $3 \mathrm{H})$.

${ }^{13}$ C NMR $\left(d_{6}\right.$-DMSO, $\left.100 \mathrm{MHz}\right) \delta: 174.1,144.6,139.5,137.4,132.7,131.5,128.9,128.8,127.2,126.9,123.9$, 120.9, 52.1, 44.1, 22.9.

HRMS (ESI, $m / z$ ): $[\mathrm{M}+\mathrm{H}]^{+}$calcd for $\mathrm{C}_{22} \mathrm{H}_{21} \mathrm{CINO}^{+}$: 350.1306; found: 350.1326 .<smiles>CC(Cc1ccc(Br)cc1)(C(=O)Nc1ccccc1)c1ccccc1</smiles>

3-(4-bromophenyl)-2-methyl- $N$,2-diphenylpropanamide (3b)

Isolated with $104 \mathrm{mg}(53 \%)$, Yellow oil.

IR 3407,3342,3052,1667,1600,1535,1495,1439,1309,1242,1110,1071,1010,757,696

${ }^{1}$ H NMR $\left(d_{6}\right.$-DMSO, $\left.500 \mathrm{MHz}\right) \delta: 9.20(\mathrm{~s}, 1 \mathrm{H}), 7.57(\mathrm{~d}, J=7.6 \mathrm{~Hz}, 2 \mathrm{H}), 7.34(\mathrm{~m}, 6 \mathrm{H}), 7.27(\mathrm{~m}, 3 \mathrm{H}), 7.04(\mathrm{t}, J=$ $7.4 \mathrm{~Hz}, 1 \mathrm{H}), 6.89$ (d, $J=8.3 \mathrm{~Hz}, 2 \mathrm{H}), 3.47$ (d, $J=13.2 \mathrm{~Hz}, 1 \mathrm{H}), 3.16$ (d, $J=13.2 \mathrm{~Hz}, 1 \mathrm{H}), 1.48$ (s, $3 \mathrm{H})$.

${ }^{13}$ C NMR $\left(d_{6}\right.$-DMSO, $\left.125 \mathrm{MHz}\right) \delta: 174.1,144.6,139.5,137.8,133.1,130.9,128.9,128.8,127.2,126.9,123.9$, 121.0, 120.0, 52.0, 44.1, 22.9.

HRMS (ESI, $m / z$ ): [M-H] $]^{-}$calcd for $\mathrm{C}_{22} \mathrm{H}_{19} \mathrm{BrNO}^{-}$: 392.0656 ; found: 392.0648 .

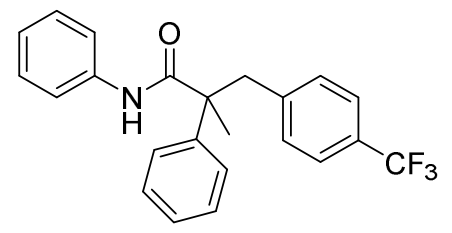

2-methyl- $N$,2-diphenyl-3-(4-(trifluoromethyl)phenyl)propanamide (3c)

Isolated with $91 \mathrm{mg}(48 \%)$, Yellow solid, m.p. $163-164^{\circ} \mathrm{C}$

IR 3412,3342,3057,1667,1601,1538,1498,1440,1325,1164,1115,1066,758,669

${ }^{1}$ H NMR $\left(d_{6}\right.$-DMSO, $\left.400 \mathrm{MHz}\right) \delta: 9.25(\mathrm{~s}, 1 \mathrm{H}), 7.58(\mathrm{~m}, 2 \mathrm{H}), 7.51(\mathrm{~d}, J=8.1 \mathrm{~Hz}, 2 \mathrm{H}), 7.34(\mathrm{~m}, 4 \mathrm{H}), 7.27(\mathrm{~m}$, $3 \mathrm{H}), 7.16(\mathrm{~d}, J=8.0 \mathrm{~Hz} .2 \mathrm{H}), 7.04(\mathrm{~m}, 1 \mathrm{H}), 3.58(\mathrm{~d}, J=13.0 \mathrm{~Hz}, 1 \mathrm{H}), 3.28(\mathrm{~d}, J=13.0 \mathrm{~Hz}, 1 \mathrm{H}), 1.48(\mathrm{~s}, 3 \mathrm{H})$, $1.50(\mathrm{~s}, 3 \mathrm{H})$.

${ }^{13}$ C NMR $\left(d_{6}\right.$-DMSO, $\left.100 \mathrm{MHz}\right) \delta: 174.1,144.5,143.5,139.5,131.8,130.9,128.9,128.8,127.6,127.3,126.9$, $126.3,124.9,124.8,124.0,123.6,121.0,52.1,44.6,22.8$.

HRMS (ESI, $m / z$ ): [M-H] $]^{-}$calcd for $\mathrm{C}_{23} \mathrm{H}_{19} \mathrm{~F}_{3} \mathrm{NO}^{-}:$: 382.1424; found: 382.1421 . 


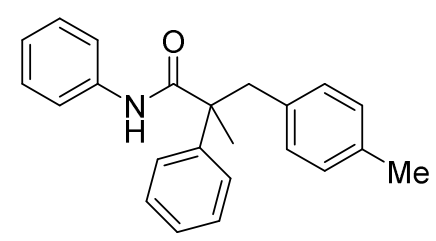

2-methyl- $N$,2-diphenyl-3-(p-tolyl)propanamide (3d)

Isolated with $75 \mathrm{mg}(\mathbf{4 6 \%})$,Yellow oil.

IR 3422,33939,3055,1667,1599,1533,1497,1438,1310,1243,1052,1027,1008,755,695

${ }^{1}$ H NMR $\left(d_{6}\right.$-DMSO, $\left.400 \mathrm{MHz}\right) \delta: 9.20(\mathrm{~s}, 1 \mathrm{H}), 7.59(\mathrm{~d}, J=8.4 \mathrm{~Hz}, 2 \mathrm{H}), 7.35(\mathrm{~m}, 3 \mathrm{H}), 7.27(\mathrm{~m}, 3 \mathrm{H}), 7.03(\mathrm{~m}$, $1 \mathrm{H}), 6.95(\mathrm{~d}, J=8.4 \mathrm{~Hz}, 2 \mathrm{H}), 6.84(\mathrm{~d}, J=8.0 \mathrm{~Hz}, 2 \mathrm{H}), 3.50(\mathrm{~d}, J=13.2 \mathrm{~Hz}, 1 \mathrm{H}), 3.14(\mathrm{~d}, J=13.2 \mathrm{~Hz}, 1 \mathrm{H}), 2.21(\mathrm{~s}$, $3 \mathrm{H}), 1.46(\mathrm{~s}, 3 \mathrm{H})$.

${ }^{13}$ C NMR $\left(d_{6}\right.$-DMSO, $\left.100 \mathrm{MHz}\right) \delta: 174.3,145.1,139.6,135.6,135.2,130.9,128.9,128.7,127.1,126.9,123.9$, $120.9,52.1,44.3,23.3,21.1$.

HRMS (ESI, $m / z$ ): [M-H] $]^{-}$calcd for $\mathrm{C}_{23} \mathrm{H}_{22} \mathrm{NO}^{-}: 328.1107$; found: 328.1700 .

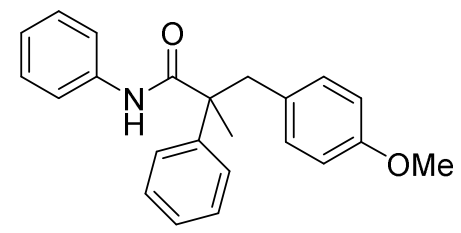

3-(4-methoxyphenyl)-2-methyl- $N, 2$-diphenylpropanamide (3e)

Isolated with $81 \mathrm{mg}(47 \%)$,Yellow solid, m.p. $180-181^{\circ} \mathrm{C}$

IR 3399,3058,1676,1601,1513,1440,1303,1244,1181,1115,1036,834,756,696

${ }^{1}$ H NMR $\left(d_{6}\right.$-DMSO, 400 MHz) $\delta: 9.19(\mathrm{~s}, 1 \mathrm{H}), 7.58(\mathrm{~m}, 2 \mathrm{H}), 7.33(\mathrm{~m}, 4 \mathrm{H}), 7.25(\mathrm{~m}, 3 \mathrm{H}), 7.04(\mathrm{~m}, 1 \mathrm{H}), 6.87(\mathrm{~d}$, $J=8.6 \mathrm{~Hz}, 2 \mathrm{H}), 6.71(\mathrm{~d}, J=8.7 \mathrm{~Hz}, 2 \mathrm{H}), 3.67(\mathrm{~s}, 3 \mathrm{H}), 3.48(\mathrm{~d}, J=13.4 \mathrm{~Hz}, 1 \mathrm{H}), 3.10(\mathrm{~d}, J=13.4 \mathrm{~Hz}, 1 \mathrm{H}), 1.45$ (s, 3H.)

${ }^{13}$ C NMR $\left(d_{6}\right.$-DMSO, $\left.100 \mathrm{MHz}\right) \delta: 174.3,158.2,145.2,139.6,131.9,130.1,128.9,128.7,127.0,126.9,123.9$, $120.9,113.5,55.4,52.2,43.8,23.2$.

HRMS (ESI, $m / z)$ : [M-H] calcd for $\mathrm{C}_{23} \mathrm{H}_{22} \mathrm{NO}_{2}^{-}: 344.1656$; found: 344.1656 .

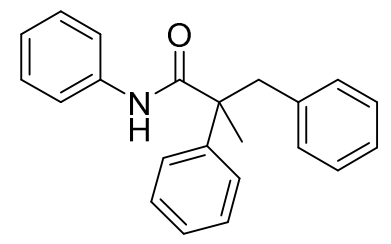

2-methyl- $N, 2,3$-triphenylpropanamide (3f)

Isolated with $71 \mathrm{mg}(\mathbf{4 5 \%})$,Yellow oil.

IR 3431,3341,3063,1662,1599,1535,1498,1439,1313,1240,1097,1077,1030,762,697

${ }^{1}$ H NMR $\left(d_{6}\right.$-DMSO, $\left.500 \mathrm{MHz}\right) \delta: 9.18(\mathrm{~s}, 1 \mathrm{H}), 7.56(\mathrm{~d}, J=7.7 \mathrm{~Hz}, 2 \mathrm{H}), 7.34(\mathrm{~m}, 4 \mathrm{H}), 7.26(\mathrm{~m}, 3 \mathrm{H}), 7.14(\mathrm{~m}$, $3 \mathrm{H}), 7.03$ (t, $J=7.4 \mathrm{~Hz}, 1 \mathrm{H}), 6.96(\mathrm{~m}, 2 \mathrm{H}), 3.53(\mathrm{~d}, J=13.2 \mathrm{~Hz}, 1 \mathrm{H}), 3.17(\mathrm{~d}, J=13.2 \mathrm{~Hz}, 1 \mathrm{H}), 1.46(\mathrm{~s}, 3 \mathrm{H})$.

${ }^{13}$ C NMR $\left(d_{6}\right.$-DMSO, $\left.125 \mathrm{MHz}\right) \delta: 174.2,145.0,139.6,138.3,130.9,128.9,128.7,128.1,127.1,126.9,126.6$, $123.9,120.9,52.1,44.7,23.2$.

HRMS (ESI, $m / z$ ): [M-H] $]^{-}$calcd for $\mathrm{C}_{22} \mathrm{H}_{20} \mathrm{NO}^{-}: 314.1550$; found: 314.1543 . 


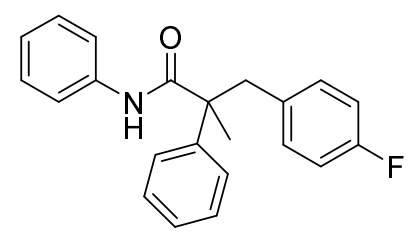

3-(4-fluorophenyl)-2-methyl- $N$,2-diphenylpropanamide (3g)

Isolated with $73 \mathrm{mg}(44 \%)$,Yellow oil.

IR 3413,3331,3057,1666,1600,1534,1506,1439,1311,1239,1222,1158,1077,1030,763,698

${ }^{1}$ H NMR $\left(d_{6}\right.$-DMSO, $\left.400 \mathrm{MHz}\right) \delta: 9.21(\mathrm{~s}, 1 \mathrm{H}), 7.57(\mathrm{~m}, 2 \mathrm{H}), 7.33(\mathrm{~m}, 7 \mathrm{H}), 7.05(\mathrm{~m}, 1 \mathrm{H}), 6.99(\mathrm{~m}, 3 \mathrm{H}), 3.49(\mathrm{~d}$, $J=13.3 \mathrm{~Hz}, 1 \mathrm{H}), 3.17(\mathrm{~d}, J=13.3 \mathrm{~Hz}, 1 \mathrm{H}), 1.48(\mathrm{~s}, 3 \mathrm{H})$.

${ }^{13}$ C NMR $\left(d_{6}\right.$-DMSO, $\left.100 \mathrm{MHz}\right) \delta: 174.2,162.6,160.2,144.8,139.5,134.5,134.3,132.7,132.6,128.9,128.8$, $127.2,126.9,123.9,120.9,114.9,114.7,52.1,43.9,22.9$.

HRMS (ESI, $m / z)$ : [M-H] calcd for $\mathrm{C}_{22} \mathrm{H}_{19} \mathrm{FNO}^{-}: 332.1456$; found: 332.1457 .

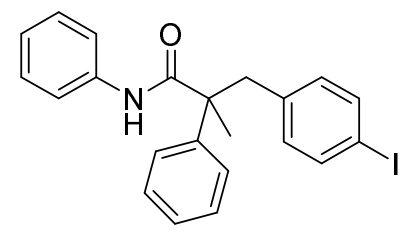

3-(4-iodophenyl)-2-methyl- $N$,2-diphenylpropanamide (3h)

Isolated with $54 \mathrm{mg}(25 \%)$,Yellow oil.

IR 3408,3343,3057,1672,1598,1526,1497,1438,1311,1244,1183,1051,1026,1006,755,697

${ }^{1}$ H NMR $\left(d_{6}\right.$-DMSO, $\left.500 \mathrm{MHz}\right) \delta: 9.18(\mathrm{~s}, 1 \mathrm{H}), 7.55(\mathrm{~d}, J=7.8 \mathrm{~Hz}, 2 \mathrm{H}), 7.50(\mathrm{~d}, J=8.2 \mathrm{~Hz}, 2 \mathrm{H}), 7.35(\mathrm{~m}, 2 \mathrm{H})$, $7.31(\mathrm{~d}, J=7.2 \mathrm{~Hz}, 1 \mathrm{H}), 7.27(\mathrm{~m}, 3 \mathrm{H}), 7.04(\mathrm{t}, J=7.3 \mathrm{~Hz}, 1 \mathrm{H}), 6.75(\mathrm{~d}, J=8.3 \mathrm{~Hz}, 2 \mathrm{H}), 3.45(\mathrm{~d}, J=13.2 \mathrm{~Hz}, 1 \mathrm{H})$, $3.13(\mathrm{~d}, J=13.2 \mathrm{~Hz}, 1 \mathrm{H}), 1.46(\mathrm{~s}, 3 \mathrm{H})$.

${ }^{13}$ C NMR $\left(d_{6}\right.$-DMSO, 125 MHz) $\delta: 174.1,144.7,139.5,138.2,136.8,133.4,128.9,128.8,127.2,126.9,123.9$, 121.0, 92.8, 52.0, 44.2, 22.9 .

HRMS (ESI, $m / z)$ : [M-H] calcd for $\mathrm{C}_{22} \mathrm{H}_{19} \mathrm{INO}^{-}: 440.0517$; found: 440.0511 .<smiles>CC(Cc1ccc([N+](=O)[O-])cc1)(C(=O)Nc1ccccc1)c1ccccc1</smiles>

2-methyl-3-(4-nitrophenyl)- $N$,2-diphenylpropanamide (3i) Isolated with $45 \mathrm{mg}(25 \%)$,Yellow oil.

IR 3407,3059,1675,1599,1518,1497,1439,1346,1313,1244,1110,1052,1027,1008,856,757,699

${ }^{1}$ H NMR $\left(d_{6}\right.$-DMSO, $\left.400 \mathrm{MHz}\right) \delta: 9.26(\mathrm{~s}, 1 \mathrm{H}), 8.02(\mathrm{~d}, J=8.8 \mathrm{~Hz}, 2 \mathrm{H}), 7.58(\mathrm{~m}, 2 \mathrm{H}), 7.34(\mathrm{~m}, 7 \mathrm{H}), 7.20(\mathrm{~d}, J=$ $8.8 \mathrm{~Hz}, 2 \mathrm{H}), 7.04(\mathrm{t}, J=7.4 \mathrm{~Hz}, 1 \mathrm{H}), 3.58(\mathrm{~d}, J=13.0 \mathrm{~Hz}, 1 \mathrm{H}), 3.33$ (d, $J=13.0 \mathrm{~Hz}, 1 \mathrm{H}), 1.53$ (s, $3 \mathrm{H})$.

${ }^{13}$ C NMR $\left(d_{6}\right.$-DMSO, $\left.100 \mathrm{MHz}\right) \delta: 174.0,147.1,146.6,144.2,139.5,132.3,128.9,128.8,127.4,126.9,124.1$, 123.1, 121.1, 52.3, 44.8, 22.7.

HRMS (ESI, $m / z)$ : [M-H] $]^{-}$calcd for $\mathrm{C}_{22} \mathrm{H}_{19} \mathrm{~N}_{2} \mathrm{O}_{3}{ }^{-}: 359.1401$; found: 359.1395 . 
<smiles>CC(Cc1cccc(Cl)c1)(C(=O)Nc1ccccc1)c1ccccc1</smiles>

3-(3-chlorophenyl)-2-methyl- $N$,2-diphenylpropanamide (3j)

Isolated with $83 \mathrm{mg}(48 \%)$,Yellow oil.

IR 3408,3245,3059,1669,1598,1536,1493,1438,1313,1248,1050,1027,758,699

${ }^{1}$ H NMR $\left(d_{6}\right.$-DMSO, $\left.400 \mathrm{MHz}\right) \delta: 9.23(\mathrm{~s}, 1 \mathrm{H}), 7.57(\mathrm{~m}, 2 \mathrm{H}), 7.30(\mathrm{~m}, 7 \mathrm{H}), 7.18(\mathrm{~m}, 2 \mathrm{H}), 7.04(\mathrm{~m}, 1 \mathrm{H}), 6.93(\mathrm{~m}$, 2H), $3.49(\mathrm{~d}, J=13.2 \mathrm{~Hz}, 1 \mathrm{H}), 3.19(\mathrm{~d}, J=13.2 \mathrm{~Hz}, 1 \mathrm{H}), 1.49$ (s, 3H).

${ }^{13}$ C NMR $\left(d_{6}\right.$-DMSO, $\left.100 \mathrm{MHz}\right) \delta: 174.1,144.6,141.0,139.5,132 . t, 130 . t, 129.8,129.7,128.9,128.8,127.2$, $126.9,126.7,124.0,121.0,52.1,44.4,22.9$.

HRMS (ESI, $m / z$ ): [M-H] $]^{-}$calcd for $\mathrm{C}_{22} \mathrm{H}_{19} \mathrm{CINO}^{-}: 348.1161$; found: 348.1168 .<smiles>CC(Cc1ccccc1Cl)(C(=O)Nc1ccccc1)c1ccccc1</smiles>

3-(2-chlorophenyl)-2-methyl- $N$,2-diphenylpropanamide (3k)

Isolated with $61 \mathrm{mg}(35 \%)$,Yellow solid, m.p. $145-146^{\circ} \mathrm{C}$.

IR 3406,3321,3059,1675,1590,1530,1498,1439,1311,1245,1052,1028,755,697

${ }^{1}$ H NMR $\left(d_{6}\right.$-DMSO, $\left.500 \mathrm{MHz}\right) \delta: 9.21(\mathrm{~s}, 1 \mathrm{H}), 7.59(\mathrm{~d}, J=7.7 \mathrm{~Hz}, 2 \mathrm{H}), 7.37(\mathrm{~m}, 3 \mathrm{H}), 7.29(\mathrm{~m}, 5 \mathrm{H}), 7.18(\mathrm{~m}$, 1H), $7.05(\mathrm{~m}, 2 \mathrm{H}), 6.76(\mathrm{~d}, J=6.8 \mathrm{~Hz}, 1 \mathrm{H}), 3.70(\mathrm{~d}, J=13.7 \mathrm{~Hz}, 1 \mathrm{H}), 3.36(\mathrm{~d}, J=13.7 \mathrm{~Hz}, 1 \mathrm{H}), 1.52(\mathrm{~s}, 3 \mathrm{H})$.

${ }^{13}$ C NMR $\left(d_{6}\right.$-DMSO, $\left.125 \mathrm{MHz}\right) \delta$ : 174.4, 144.3, 139.5, 136.0, 135.2, 132.3, 129.7, 128.9, 128.8, 128.6, 127.3, $127.0,126.9,124.0,121.0,52.6,40.9,21.8$.

HRMS (ESI, $m / z$ ): [M-H] $]^{-}$calcd for $\mathrm{C}_{22} \mathrm{H}_{19} \mathrm{CINO}^{-}: 348.1161$; found: 348.1158 .

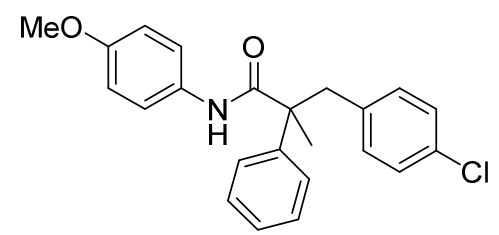

3-(4-chlorophenyl)- $N$-(4-methoxyphenyl)-2-methyl-2-phenylpropanamide (3I)

Isolated with $117 \mathrm{mg}(62 \%)$, Yellow solid, m.p. $155-156^{\circ} \mathrm{C}$.

IR 3409,3346,3051,1658,1600,1532,1511,1464,1409,1304,1233,1180,1111,1033,834,700

${ }^{1}$ H NMR $\left(d_{6}\right.$-DMSO, $\left.500 \mathrm{MHz}\right) \delta: 9.10(\mathrm{~s}, 1 \mathrm{H}), 7.45(\mathrm{~d}, J=9.0 \mathrm{~Hz}, 2 \mathrm{H}), 7.32(\mathrm{~m}, 4 \mathrm{H}), 7.24(\mathrm{~m}, 1 \mathrm{H}), 7.20(\mathrm{~d}, J=$ $8.4 \mathrm{~Hz}, 2 \mathrm{H}), 6.96(\mathrm{~d}, J=8.4 \mathrm{~Hz}, 2 \mathrm{H}), 6.85(\mathrm{~d}, J=9.0 \mathrm{~Hz}, 2 \mathrm{H}), 3.70(\mathrm{~s}, 3 \mathrm{H}), 3.47(\mathrm{~d}, J=13.2 \mathrm{~Hz}, 1 \mathrm{H}), 3.16(\mathrm{~d}, J=$ $13.2 \mathrm{~Hz}, 1 \mathrm{H}), 1.46(\mathrm{~s}, 3 \mathrm{H})$.

${ }^{13}$ C NMR $\left(d_{6}\right.$-DMSO, $\left.125 \mathrm{MHz}\right) \delta: 173.8,155.9,144.9,137.5,132.8,132.5,128.8,128.0,127.1,126.9,122.7$, 114.1, 55.6, 51.9, 44.2, 22.9.

HRMS (ESI, $m / z$ ): [M-H] $]^{-}$calcd for $\mathrm{C}_{23} \mathrm{H}_{21} \mathrm{ClNO}_{2}^{-}: 378.1266$; found: 378.1256 . 
<smiles>Cc1ccc(NC(=O)C(C)(Cc2ccc(Cl)cc2)c2ccccc2)cc1</smiles>

3-(4-chlorophenyl)-2-methyl-2-phenyl- $N$-(p-tolyl)propanamide (3m)

Isolated with $110 \mathrm{mg}(61 \%)$,Yellow oil

IR 3409,3298,3055,1673,1598,1516,1492,1404,1312,1244,1093,1053,1027,1014,818,701

${ }^{1}$ H NMR $\left(d_{6}\right.$-DMSO, $\left.500 \mathrm{MHz}\right) \delta: 9.13(\mathrm{~s}, 1 \mathrm{H}), 7.45(\mathrm{~d}, J=8.4 \mathrm{~Hz}, 2 \mathrm{H}), 7.33(\mathrm{~m}, 4 \mathrm{H}), 7.26(\mathrm{t}, J=7.0 \mathrm{~Hz}, 1 \mathrm{H})$, $7.2(\mathrm{~d}, J=8.4 \mathrm{~Hz}, 2 \mathrm{H}), 7.07(\mathrm{~d}, J=8.3 \mathrm{~Hz}, 2 \mathrm{H}), 6.95(\mathrm{~d}, J=8.3 \mathrm{~Hz}, 2 \mathrm{H}), 3.48(\mathrm{~d}, J=13.2 \mathrm{~Hz}, 1 \mathrm{H}), 3.16(\mathrm{~d}, J=$ $13.2 \mathrm{~Hz}, 1 \mathrm{H}), 2.23(\mathrm{~s}, 3 \mathrm{H}), 1.47(\mathrm{~s}, 3 \mathrm{H})$.

${ }^{13}$ C NMR $\left(d_{6}\right.$-DMSO, $\left.125 \mathrm{MHz}\right) \delta: 173.9,144.8,137.5,136.9,132.9,132.8,129.3,128.8,128.0,127.2,126.9$, 121.0, 52.0, 44.1, 22.9, 20.9.

HRMS (ESI, $m / z)$ : [M-H] $]^{-}$calcd for $\mathrm{C}_{23} \mathrm{H}_{21} \mathrm{CINO}^{-}: 362.1317$; found: 362.1321 .

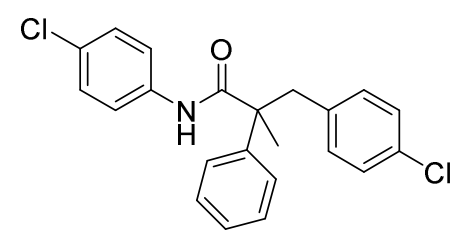

N,3-bis(4-chlorophenyl)-2-methyl-2-phenylpropanamide (3n)

Isolated with $103 \mathrm{mg}(54 \%)$, Yellow solid, m.p. 96-97 ${ }^{\circ} \mathrm{C}$.

IR 3410,3252,3052,1676,1595,1525,1492,1396,1303,1244,1090,1054,1026,1011,823,699

${ }^{1}$ H NMR $\left(d_{6}\right.$-DMSO, $\left.500 \mathrm{MHz}\right) \delta: 9.36(\mathrm{~s}, 1 \mathrm{H}), 7.64(\mathrm{~d}, J=8.9 \mathrm{~Hz}, 2 \mathrm{H}), 7.32(\mathrm{~m}, 7 \mathrm{H}), 7.20(\mathrm{~d}, J=8.3 \mathrm{~Hz}, 2 \mathrm{H})$, $6.93(\mathrm{~d}, J=8.3 \mathrm{~Hz}, 2 \mathrm{H}), 3.47$ (d, $J=13.2 \mathrm{~Hz}, 1 \mathrm{H}), 3.18(\mathrm{~d}, J=13.2 \mathrm{~Hz}, 1 \mathrm{H}), 1.48(\mathrm{~s}, 3 \mathrm{H})$.

${ }^{13}$ C NMR $\left(d_{6}\right.$-DMSO, 125 MHz) $\delta: 174.3,144.4,138.5,137.3,132.7,128.8,128.7,128.0,127.3,126.9,122.4$, $52.2,44.1,22.8$.

HRMS (ESI, $m / z)$ : [M-H] $]^{-}$calcd for $\mathrm{C}_{22} \mathrm{H}_{19} \mathrm{Cl}_{2} \mathrm{NO}^{-}: 382.0771$; found: 382.0765 .

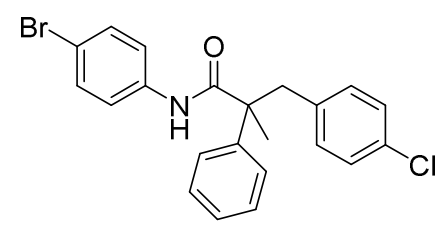

N-(4-bromophenyl)-3-(4-chlorophenyl)-2-methyl-2-phenylpropanamide (3o)

Isolated with $123 \mathrm{mg}(55 \%)$,Yellow oil.

IR 3408,3250,3057,1678,1590,1526,1489,1392,1304,1241,1026,1008,825,700

${ }^{1}$ H NMR $\left(d_{6}\right.$-DMSO, $\left.400 \mathrm{MHz}\right) \delta: 9.36(\mathrm{~s}, 1 \mathrm{H}), 7.58(\mathrm{~d}, J=9.0 \mathrm{~Hz}, 2 \mathrm{H}), 7.45(\mathrm{~d}, J=8.9 \mathrm{~Hz}, 2 \mathrm{H}), 7.35(\mathrm{~m}, 2 \mathrm{H})$, $7.28(\mathrm{~m}, 3 \mathrm{H}), 7.19(\mathrm{~d}, J=8.4 \mathrm{~Hz}, 2 \mathrm{H}), 6.92(\mathrm{~d}, J=8.4 \mathrm{~Hz}, 2 \mathrm{H}), 3.46(\mathrm{~d}, J=13.2 \mathrm{~Hz}, 1 \mathrm{H}), 3.18(\mathrm{~d}, J=13.2 \mathrm{~Hz}$, $1 \mathrm{H}), 1.47(\mathrm{~s}, 3 \mathrm{H})$.

${ }^{13}$ C NMR $\left(d_{6}\right.$-DMSO, $\left.100 \mathrm{MHz}\right) \delta: 174.3,144.4,138.9,137.2,132.7,131.8,128.8,128.0,127.3,126.9,122.8$, 115.6, 52.2, 44.0, 22.8.

HRMS (ESI, $m / z$ ): $[\mathrm{M}+\mathrm{Na}]^{+}$calcd for $\mathrm{C}_{22} \mathrm{H}_{19} \mathrm{BrClNONa}^{+}: 450.0231$; found: 450.0219 . 


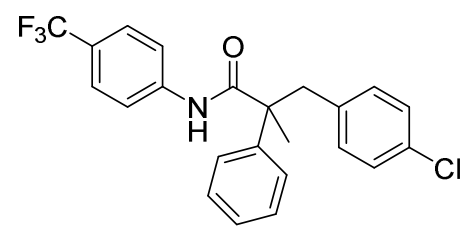

3-(4-chlorophenyl)-2-methyl-2-phenyl- $N$-(4-(trifluoromethyl)phenyl)propanamide (3p)

Isolated with $97 \mathrm{mg}(\mathbf{4 7 \%})$,Yellow oil.

IR 3409,3058,1658,1602,1526,1492,1407,1324,1252,1164,1116,1052,1015,842,761,700

${ }^{1}$ H NMR $\left(d_{6}\right.$-DMSO, $\left.400 \mathrm{MHz}\right) \delta: 9.59(\mathrm{~s}, 1 \mathrm{H}), 7.85(\mathrm{~d}, J=8.5 \mathrm{~Hz}, 2 \mathrm{H}), 7.64(\mathrm{~d}, J=8.7 \mathrm{~Hz}, 2 \mathrm{H}), 7.35(\mathrm{~m}, 2 \mathrm{H})$, $7.28(\mathrm{~m}, 2 \mathrm{H}), 7.19(\mathrm{~d}, J=8.4 \mathrm{~Hz}, 2 \mathrm{H}), 6.92(\mathrm{~d}, J=8.4 \mathrm{~Hz}, 2 \mathrm{H}), 3.47(\mathrm{~d}, J=13.2 \mathrm{~Hz}, 1 \mathrm{H}), 3.21(\mathrm{~d}, J=13.2 \mathrm{~Hz}$, $1 \mathrm{H}), 1.50(\mathrm{~s}, 3 \mathrm{H})$.

${ }^{13}$ C NMR $\left(d_{6}\right.$-DMSO, $\left.100 \mathrm{MHz}\right) \delta: 174.8,144.2,143.2,137.2,132.7,131.6,128.9,128.1,127.3,127.0,126.3$, 126.2, 124.3, 124.1, 123.8, 123.5, 120.6, 52.4, 44.0, 22.8 .

HRMS (ESI, $m / z)$ : [M-H] calcd for $\mathrm{C}_{23} \mathrm{H}_{19} \mathrm{ClF}_{3} \mathrm{NO}^{-}$: 416.1035 ; found: 416.1024 .

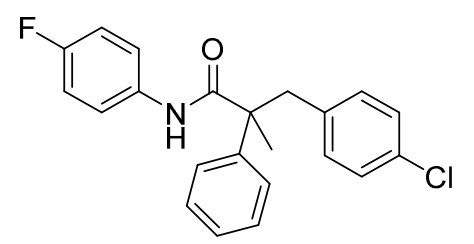

3-(4-chlorophenyl)- $N$-(4-fluorophenyl)-2-methyl-2-phenylpropanamide (3q) Isolated with $80 \mathrm{mg}(44 \%)$,Yellow oil.

IR 3410,3274,3059,1675,1611,1534,1509,1492,1404,1306,1213,1093,1027,1012,838,701

${ }^{1}$ H NMR $\left(d_{6}\right.$-DMSO, $\left.400 \mathrm{MHz}\right) \delta: 9.29(\mathrm{~s}, 1 \mathrm{H}), 7.59(\mathrm{~m}, 2 \mathrm{H}), 7.33(\mathrm{~m}, 4 \mathrm{H}), 7.26(\mathrm{~m}, 1 \mathrm{H}), 7.20(\mathrm{~d}, J=8.4 \mathrm{~Hz}$, $2 \mathrm{H}), 7.11(\mathrm{t}, J=8.9 \mathrm{~Hz}, 2 \mathrm{H}), 6.94(\mathrm{~d}, J=8.4 \mathrm{~Hz}, 2 \mathrm{H}), 3.46(\mathrm{~d}, J=13.2 \mathrm{~Hz}, 1 \mathrm{H}), 3.17(\mathrm{~d}, J=13.2 \mathrm{~Hz}, 1 \mathrm{H}), 1.47(\mathrm{~s}$, $3 \mathrm{H})$.

${ }^{13}$ C NMR $\left(d_{6}\right.$-DMSO, $\left.100 \mathrm{MHz}\right) \delta: 174.1,159.9,157.5,144.6,137.4,135.9,132.8,131.5,128.8,128.1,127.2$, $126.9,122.8,122.8,115.6,115.4,52.0,44.1,22.9$.

HRMS (ESI, $m / z)$ : [M-H] $]^{-}$calcd for $\mathrm{C}_{22} \mathrm{H}_{19} \mathrm{ClFNO}^{-}: 366.1066$; found: 366.1061 .

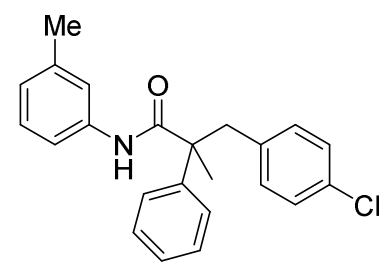

3-(4-chlorophenyl)-2-methyl-2-phenyl-N-(m-tolyl)propanamide (3r) Isolated with $92 \mathrm{mg}(51 \%)$,Yellow oil.

IR 3409,3336,3058,1673,1595,1538,1490,1305,1255,1093,1052,1027,817,780,699

${ }^{1}$ H NMR $\left(d_{6}\right.$-DMSO, $\left.500 \mathrm{MHz}\right) \delta: 9.11(\mathrm{~s}, 1 \mathrm{H}), 7.35(\mathrm{~m}, 6 \mathrm{H}), 7.26(\mathrm{t}, J=7 \mathrm{~Hz}, 1 \mathrm{H}), 7.21(\mathrm{~d}, J=8.4 \mathrm{~Hz}, 2 \mathrm{H}), 7.15$ $(\mathrm{m}, 1 \mathrm{H}), 6.95(\mathrm{~d}, J=8.4 \mathrm{~Hz}, 2 \mathrm{H}), 6.85(\mathrm{~d}, J=7.4 \mathrm{~Hz}, 1 \mathrm{H}), 3.48(\mathrm{~d}, J=13.2 \mathrm{~Hz}, 1 \mathrm{H}), 3.17(\mathrm{~d}, J=13.2 \mathrm{~Hz}, 1 \mathrm{H})$, $2.25(\mathrm{~s}, 3 \mathrm{H}), 1.47(\mathrm{~s}, 3 \mathrm{H})$.

${ }^{13}$ C NMR $\left(d_{6}\right.$-DMSO, $\left.125 \mathrm{MHz}\right) \delta: 174.1,144.6,139.4,138.1,137.4,132.7,131.5,128.7,128.0,127.2,126.9$, 124.6, 121.4, 118.0, 52.0, 44.0, 22.8, 21.6.

HRMS (ESI, $m / z$ ): [M-H] $]^{-}$calcd for $\mathrm{C}_{23} \mathrm{H}_{21} \mathrm{CINO}^{-}: 362.1317$; found: 362.1313 . 


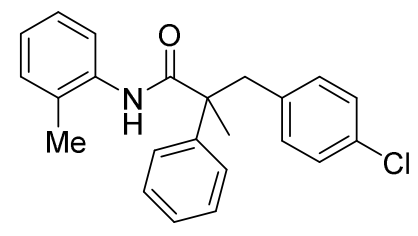

3-(4-chlorophenyl)-2-methyl-2-phenyl- $N$-(o-tolyl)propanamide (3s)

Isolated with $70 \mathrm{mg}(39 \%)$,Yellow oil.

IR 3420,3319,3058,1671,1587,1517,1491,1451,1295,1254,1093,1052,1027,754,701

${ }^{1}$ H NMR $\left(d_{6}\right.$-DMSO, $\left.500 \mathrm{MHz}\right) \delta: 8.73(\mathrm{~s}, 1 \mathrm{H}), 7.38(\mathrm{~m}, 4 \mathrm{H}), 7.30(\mathrm{~m}, 1 \mathrm{H}), 7.23(\mathrm{~d}, J=8.2 \mathrm{~Hz}, 2 \mathrm{H}), 7.16(\mathrm{~m}$, 3H), $7.09(\mathrm{~m}, 1 \mathrm{H}), 6.98(\mathrm{~d}, J=8.1 \mathrm{~Hz}, 2 \mathrm{H}), 3.46(\mathrm{~d}, J=13.2 \mathrm{~Hz}, 1 \mathrm{H}), 3.16(\mathrm{~d}, J=13.2 \mathrm{~Hz}, 1 \mathrm{H}), 1.95(\mathrm{~s}, 3 \mathrm{H})$, $1.52(\mathrm{~s}, 3 \mathrm{H})$.

${ }^{13}$ C NMR $\left(d_{6}\right.$-DMSO, $\left.125 \mathrm{MHz}\right) \delta: 174.2,144.6,137.5,136.8,133.9,132.8,131.4,130.6,128.7,128.0,127.2$, $127.0,126.9,126.3,126.2,51.5,44.2,22.6,17.9$.

HRMS (ESI, $m / z)$ : [M-H] $]^{-}$calcd for $\mathrm{C}_{23} \mathrm{H}_{21} \mathrm{CINO}^{-}: 362.1317$; found: 362.1313 .

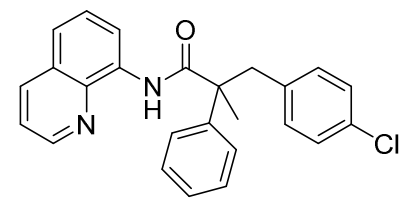

3-(4-chlorophenyl)-2-methyl-2-phenyl- $N$-(quinolin-8-yl)propanamide (3t)

Isolated with $86 \mathrm{mg}(43 \%)$,Yellow solid, m.p. $125-126^{\circ} \mathrm{C}$.

IR 3440,3347,3057,1681,1597,1524,1486,1424,1384,1326,1094,1015,827,792,702

${ }^{1}$ H NMR $\left(d_{6}\right.$-DMSO, $\left.500 \mathrm{MHz}\right) \delta: 9.77(\mathrm{~s}, 1 \mathrm{H}), 8.68(\mathrm{~m}, 1 \mathrm{H}), 8.65(\mathrm{~d}, J=6.8 \mathrm{~Hz}, 1 \mathrm{H}), 8.36(\mathrm{~m}, 1 \mathrm{H}), 7.65(\mathrm{~d}, J=$ $7.5 \mathrm{~Hz}, 1 \mathrm{H}), 7.59$ (d, $J=7.8 \mathrm{~Hz}, 1 \mathrm{H}), 7.56(\mathrm{~m}, 1 \mathrm{H}), 7.44(\mathrm{~d}, J=7.5 \mathrm{~Hz}, 2 \mathrm{H}), 7.39$ (m, 2H), $7.32(\mathrm{t}, J=7.1 \mathrm{~Hz}$, $1 \mathrm{H}), 7.18(\mathrm{~d}, J=8.3 \mathrm{~Hz}, 2 \mathrm{H}), 6.92(\mathrm{~d}, J=8.3 \mathrm{~Hz}, 2 \mathrm{H}), 3.48(\mathrm{~d}, J=13.2 \mathrm{~Hz}, 1 \mathrm{H}), 3.33(\mathrm{~d}, J=13.2 \mathrm{~Hz}, 1 \mathrm{H}), 1.59$ $(\mathrm{s}, 3 \mathrm{H})$.

${ }^{13}$ C NMR $\left(d_{6}\right.$-DMSO, $\left.125 \mathrm{MHz}\right) \delta: 174.2,149.5,143.3,137.2,136.9,132.7,129.2,128.2,128.1,127.8,127.5$, $127.3,122.7,122.3,115.9,52.8,44.0,23.0$.

HRMS (ESI, $m / z)$ : $[\mathrm{M}+\mathrm{H}]^{+}$calcd for $\mathrm{C}_{25} \mathrm{H}_{22} \mathrm{ClN}_{2} \mathrm{O}^{+}: 401.1415$; found: 401.1430 .

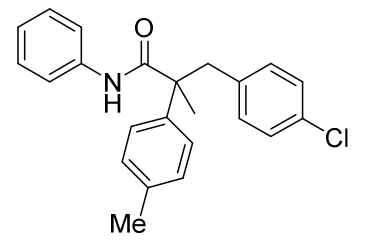

3-(4-chlorophenyl)-2-methyl- $N$-phenyl-2-(p-tolyl)propanamide (3u)

Isolated with $114 \mathrm{mg}(63 \%)$,Yellow solid, m.p. $161-162^{\circ} \mathrm{C}$.

IR 3406,3320,3052,1667,1597,1532,1496,1439,1308,1243,1093,1014,753,693

${ }^{1}$ H NMR $\left(d_{6}\right.$-DMSO, $\left.500 \mathrm{MHz}\right) \delta: 9.16(\mathrm{~s}, 1 \mathrm{H}), 7.60(\mathrm{~d}, J=7.9 \mathrm{~Hz}, 2 \mathrm{H}), 7.27(\mathrm{~m}, 2 \mathrm{H}), 7.20(\mathrm{~m}, 4 \mathrm{H}), 7.16(\mathrm{~d}, J=$ $7.9 \mathrm{~Hz}, 2 \mathrm{H}), 7.02$ (t, $J=7.3 \mathrm{~Hz}, 1 \mathrm{H}), 6.97$ (d, $J=8.2 \mathrm{~Hz}, 2 \mathrm{H}), 3.49$ (d, J=13.2 Hz, 1H), $3.16(\mathrm{~d}, J=13.2 \mathrm{~Hz}, 1 \mathrm{H})$, $2.29(\mathrm{~s}, 3 \mathrm{H}), 1.47(\mathrm{~s}, 3 \mathrm{H})$.

${ }^{13}$ C NMR $\left(d_{6}\right.$-DMSO, 125 MHz) $\delta: 174.3,141.6,139.6,137.5,136.2,132.7,131.5,129.3,128.9,128.0,126.8$, $123.9,120.9,51.7,44.1,22.9,21.1$.

HRMS (ESI, $m / z$ ): [M-H] $]^{-}$calcd for $\mathrm{C}_{23} \mathrm{H}_{21} \mathrm{CINO}^{-}: 362.1317$; found: 362.1322 . 


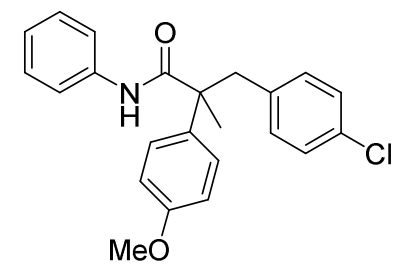

3-(4-chlorophenyl)-2-(4-methoxyphenyl)-2-methyl- $N$-phenylpropanamide (3v)

Isolated with $111 \mathrm{mg}(59 \%)$,Yellow oil.

IR 3403,3294,3058,1675,1599,1513,1493,1439,1309,1250,1185,1093,1077,1029,757,695

${ }^{1}$ H NMR $\left(d_{6}\right.$-DMSO, 400 MHz) $\delta: 9.12(\mathrm{~s}, 1 \mathrm{H}), 7.57(\mathrm{~m}, 2 \mathrm{H}), 7.27(\mathrm{~m}, 2 \mathrm{H}), 7.22(\mathrm{~m}, 4 \mathrm{H}), 7.03(\mathrm{~m}, 1 \mathrm{H}), 6.94(\mathrm{~d}$, $J=8.4 \mathrm{~Hz}, 2 \mathrm{H}), 6.91(\mathrm{~d}, J=8.8 \mathrm{~Hz}, 2 \mathrm{H}), 3.74(\mathrm{~s}, 3 \mathrm{H}), 3.44(\mathrm{~d}, J=13.2 \mathrm{~Hz}, 1 \mathrm{H}), 3.14(\mathrm{~d}, J=13.2 \mathrm{~Hz}, 1 \mathrm{H}), 1.45$ $(\mathrm{s}, 3 \mathrm{H})$.

${ }^{13}$ C NMR $\left(d_{6}\right.$-DMSO, $\left.100 \mathrm{MHz}\right) \delta: 174.4,158.4,139.6,137.5,132.7,131.4,128.9,128.9,128.1,128.0,123.9$, $120.9,114.1,55.5,51.3,44.3,22.8$.

HRMS (ESI, $m / z$ ): [M-H] $]^{-}$calcd for $\mathrm{C}_{23} \mathrm{H}_{21} \mathrm{ClNO}_{2}^{-}$: 378.1266; found: 378.1256 .

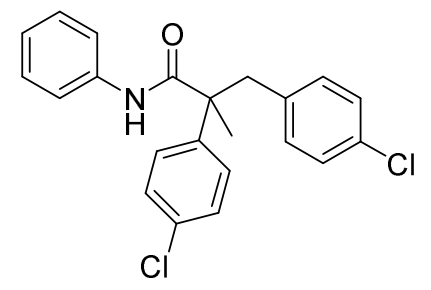

2,3-bis(4-chlorophenyl)-2-methyl- $N$-phenylpropanamide (3w)

Isolated with $112 \mathrm{mg}(59 \%)$, Yellow oil.

IR 3410,3290,3058,1676,1598,1534,1493,1439,1312,1245,1094,1028,821,727,696

${ }^{1}$ H NMR $\left(d_{6}\right.$-DMSO, $\left.400 \mathrm{MHz}\right) \delta: 9.22(\mathrm{~s}, 1 \mathrm{H}), 7.56(\mathrm{~m}, 2 \mathrm{H}), 7.41(\mathrm{~d}, J=8.6 \mathrm{~Hz}, 2 \mathrm{H}), 7.26(\mathrm{~m}, 6 \mathrm{H}), 7.04(\mathrm{~m}$, $1 \mathrm{H}), 6.95(\mathrm{~d}, J=8.4 \mathrm{~Hz}, 2 \mathrm{H}), 3.44$ (d, $J=13.2 \mathrm{~Hz}, 1 \mathrm{H}), 3.17$ (d, $J=13.2 \mathrm{~Hz}, 1 \mathrm{H}), 1.48$ (s, 3H).

${ }^{13}$ C NMR $\left(d_{6}\right.$-DMSO, $\left.100 \mathrm{MHz}\right) \delta: 173.8,143.4,139.4,137.1,132.7,131.6,129.0,128.9,128.6,128.1,124.1$, 121.0, 51.8, 44.0, 22.7.

HRMS (ESI, $m / z$ ): [M-H] $]^{-}$calcd for $\mathrm{C}_{22} \mathrm{H}_{18} \mathrm{Cl}_{2} \mathrm{NO}^{-}$: 382.0771 ; found: 382.0763 .

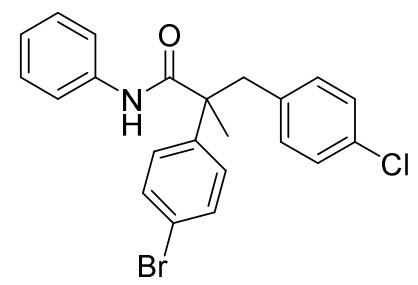

2-(4-bromophenyl)-3-(4-chlorophenyl)-2-methyl- $N$-phenylpropanamide (3x)

Isolated with 130mg (61\%), Yellow oil.

IR $3410,3291,3058,1677,1598,1492,1439,1312,1245,1092,1027,821,757,695$

${ }^{1}$ H NMR $\left(d_{6}\right.$-DMSO, $\left.400 \mathrm{MHz}\right) \delta: 9.22(\mathrm{~s}, 1 \mathrm{H}), 7.55(\mathrm{~m}, 4 \mathrm{H}), 7.25(\mathrm{~m}, 6 \mathrm{H}), 7.04(\mathrm{~m}, 1 \mathrm{H}), 6.95(\mathrm{~d}, J=8.4 \mathrm{~Hz}$, 2H), $3.44(\mathrm{~d}, J=13.2 \mathrm{~Hz}, 1 \mathrm{H}), 3.17(\mathrm{~d}, \mathrm{~J}=13.2 \mathrm{~Hz}, 1 \mathrm{H}), 1.47$ (s, 3H).

${ }^{13}$ C NMR $\left(d_{6}\right.$-DMSO, $\left.100 \mathrm{MHz}\right) \delta: 173.7,143.9,139.4,137.1,132.7,131.6,129.4,128.9,128.1,124.1,121.0$, $120.4,51.9,43.9,22.7$.

HRMS (ESI, $m / z)$ : [M-H] calcd for $\mathrm{C}_{22} \mathrm{H}_{18} \mathrm{BrClNO}^{-}$: 426.0266 ; found: 426.0294. 
<smiles>O=C(Nc1ccccc1)C(Cc1ccc(Cl)cc1)c1ccccc1</smiles>

3-(4-chlorophenyl)- $N$,2-diphenylpropanamide (3y)

Isolated with $45 \mathrm{mg}(27 \%)$, Yellow oil.

IR 3409,3303,3063,1666,1600,1546,1493,1442,1313,1250,1093,1050,1026,1011,820,757,697

${ }^{1}$ H NMR $\left(d_{6}\right.$-DMSO, $\left.400 \mathrm{MHz}\right) \delta: 10.06(\mathrm{~s}, 1 \mathrm{H}), 7.52(\mathrm{~m}, 2 \mathrm{H}), 7.43(\mathrm{~d}, J=7.4 \mathrm{~Hz}, 2 \mathrm{H}), 7.30(\mathrm{~m}, 9 \mathrm{H}), 6.99(\mathrm{t}, J=$ $7.4 \mathrm{~Hz}, 1 \mathrm{H}), 3.99(\mathrm{~m}, 1 \mathrm{H}), 3.40(\mathrm{~m}, 1 \mathrm{H}), 2.97(\mathrm{~m}, 1 \mathrm{H})$.

${ }^{13}$ C NMR $\left(d_{6}\right.$-DMSO, $\left.100 \mathrm{MHz}\right) \delta: 171.2,140.4,139.5,139.2,131.3,131.2,129.2,128.9,128.6,128.3,123.8$, $119.6,54.3,38.5$.

HRMS (ESI, $m / z$ ): [M-H] $]^{-}$calcd for $\mathrm{C}_{21} \mathrm{H}_{17} \mathrm{CINO}^{-}: 334.1004$; found: 334.1004 .

\section{Control Experiments}

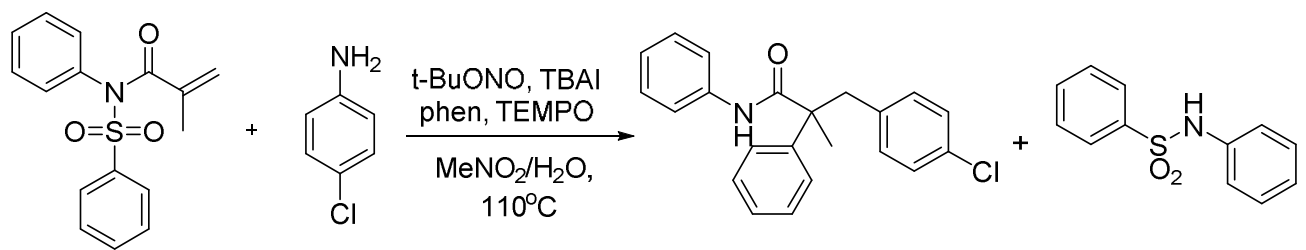

In a dry $25 \mathrm{~mL}$ oven-dried schlenk tubes, $N$-phenyl- $N$-(phenylsulfonyl)methacrylamide $(0.5 \mathrm{mmol})$, aniline (1.5 mmol, 3.0 equiv), TBAI (0.05 mmol, 0.1 equiv), phenanthroline (0.5 mmol, 1 equiv), TEMPO (1.5 mmol, 3.0 equiv) were dissolved in the mixture of $1 \mathrm{~mL} \mathrm{MeNO}$ and $50 \mu \mathrm{L} \mathrm{H}_{2} \mathrm{O}$ under an atmosphere of dry nitrogen at room temperature. Tert-butyl nitrite $(1.75 \mathrm{mmol}, 3.5$ equiv) was then added with a syringe. The reaction mixture was heated to $110{ }^{\circ} \mathrm{C}$ for $10 \mathrm{~h}$. Then the solution was cooled to r.t., washed with a saturated aqueous solution of $\mathrm{NaCl}$, extracted by ethyl acetate and dried over by $\mathrm{Na}_{2} \mathrm{SO}_{4}$. The product was purified by silica gel column chromatography. 


\section{NMR Spectra}

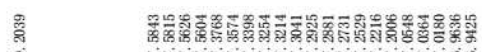

i)

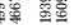

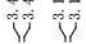

$\underset{i}{\stackrel{x}{8}}$

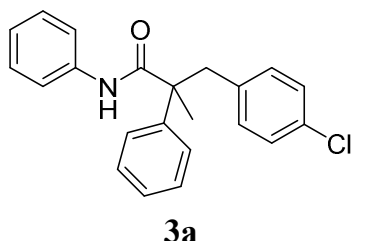

3a
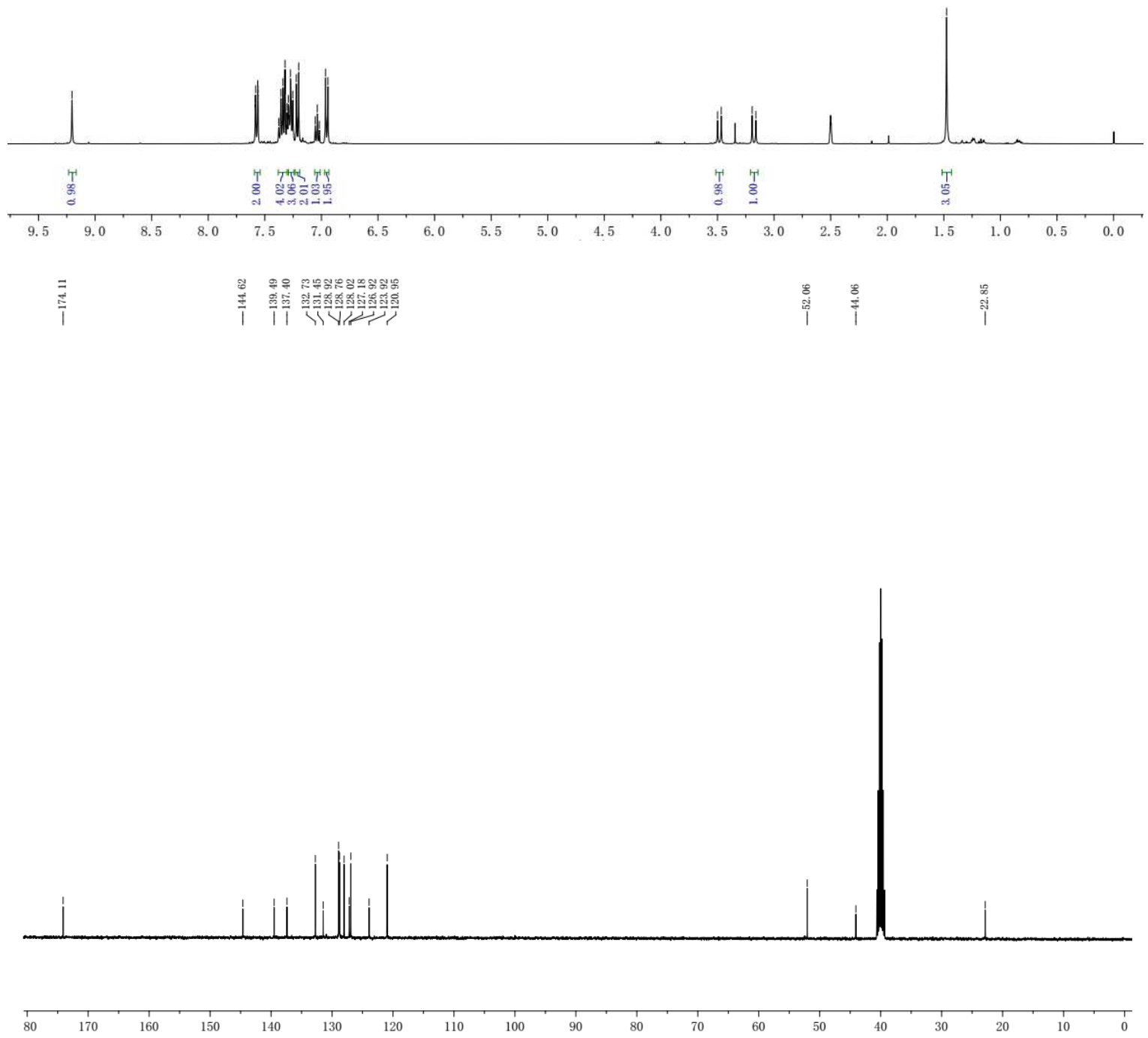

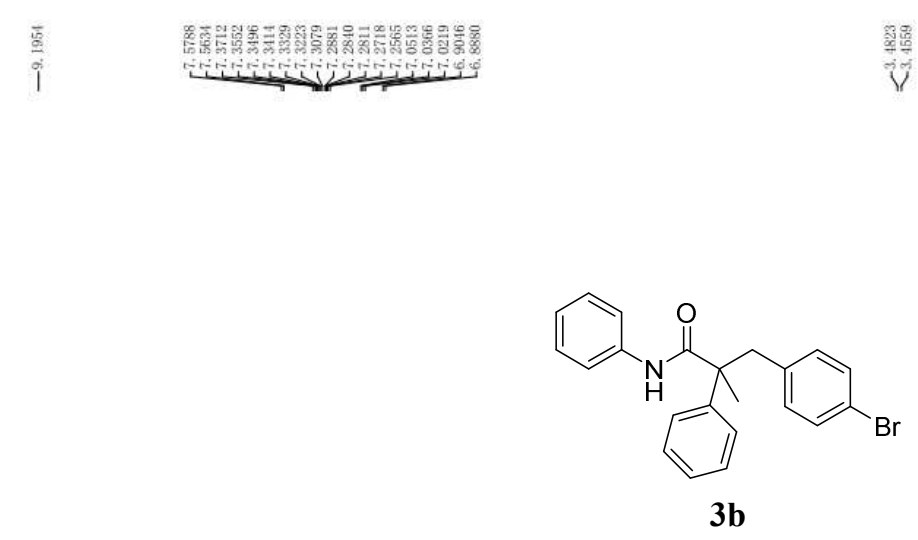

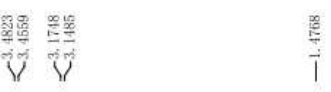
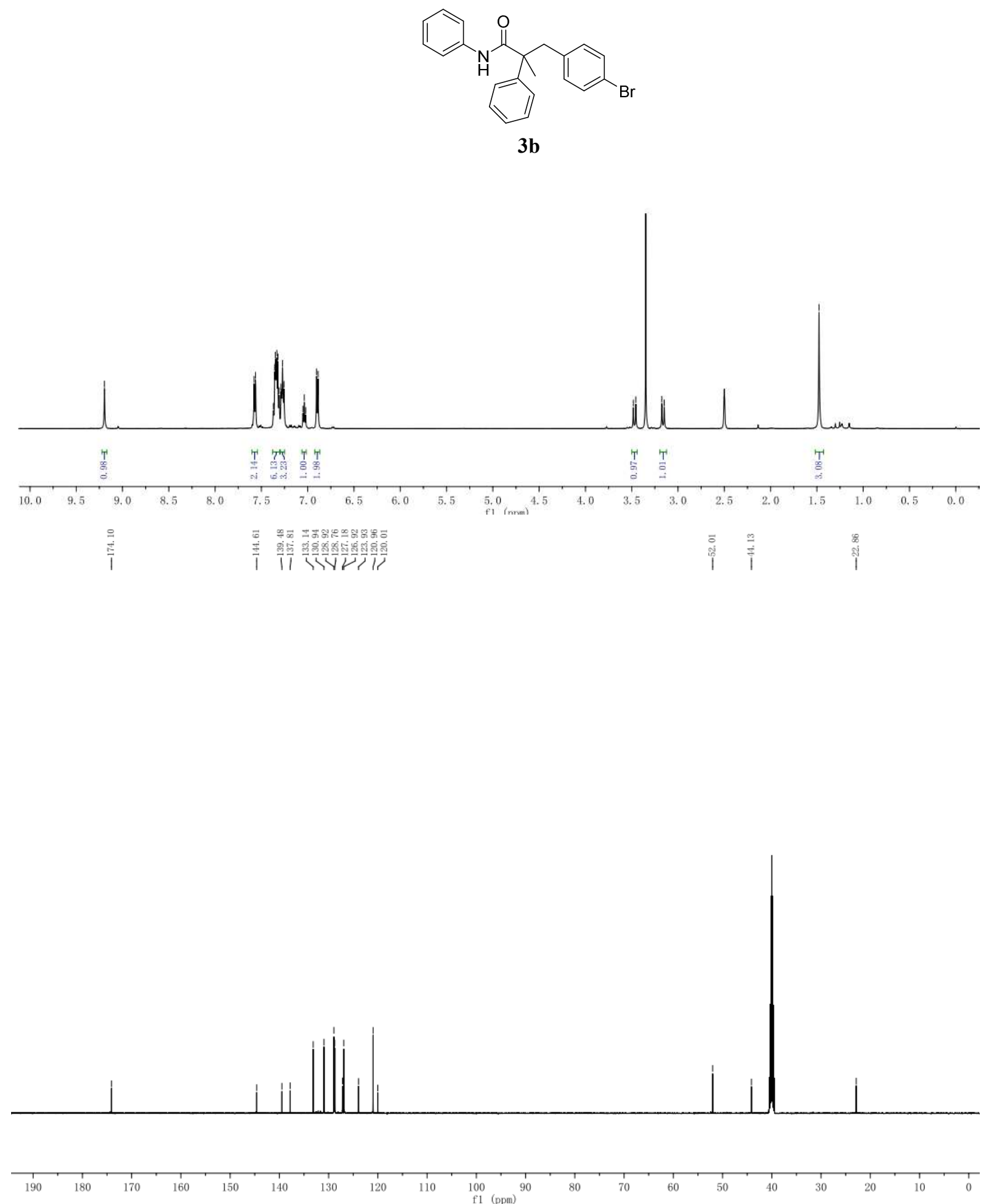
高

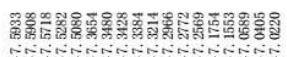

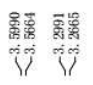

$\stackrel{\text { : }}{i}$
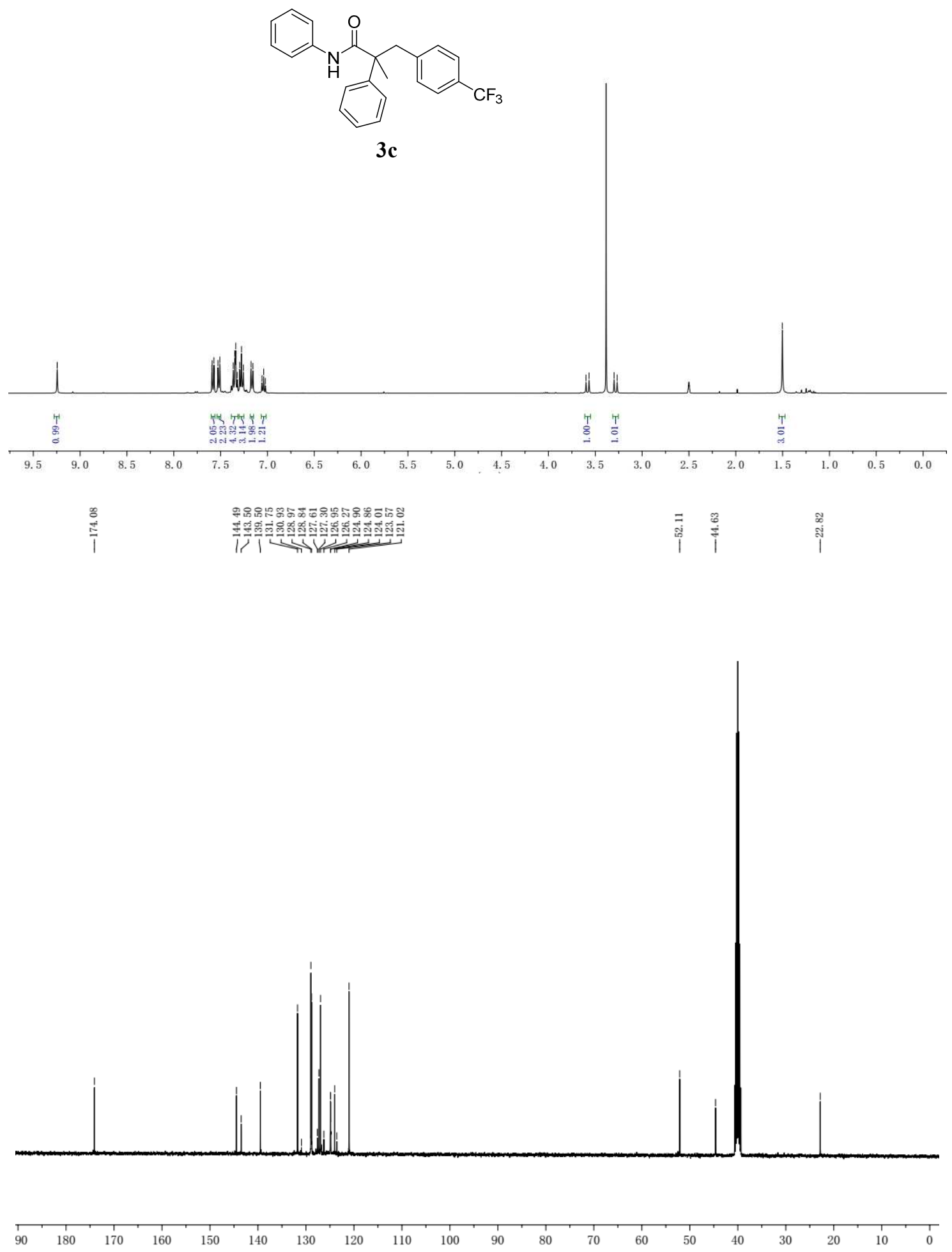

14 


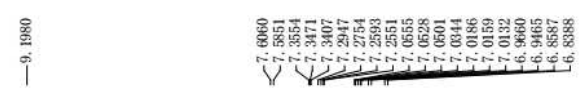

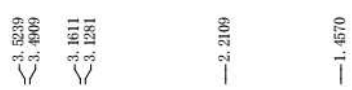
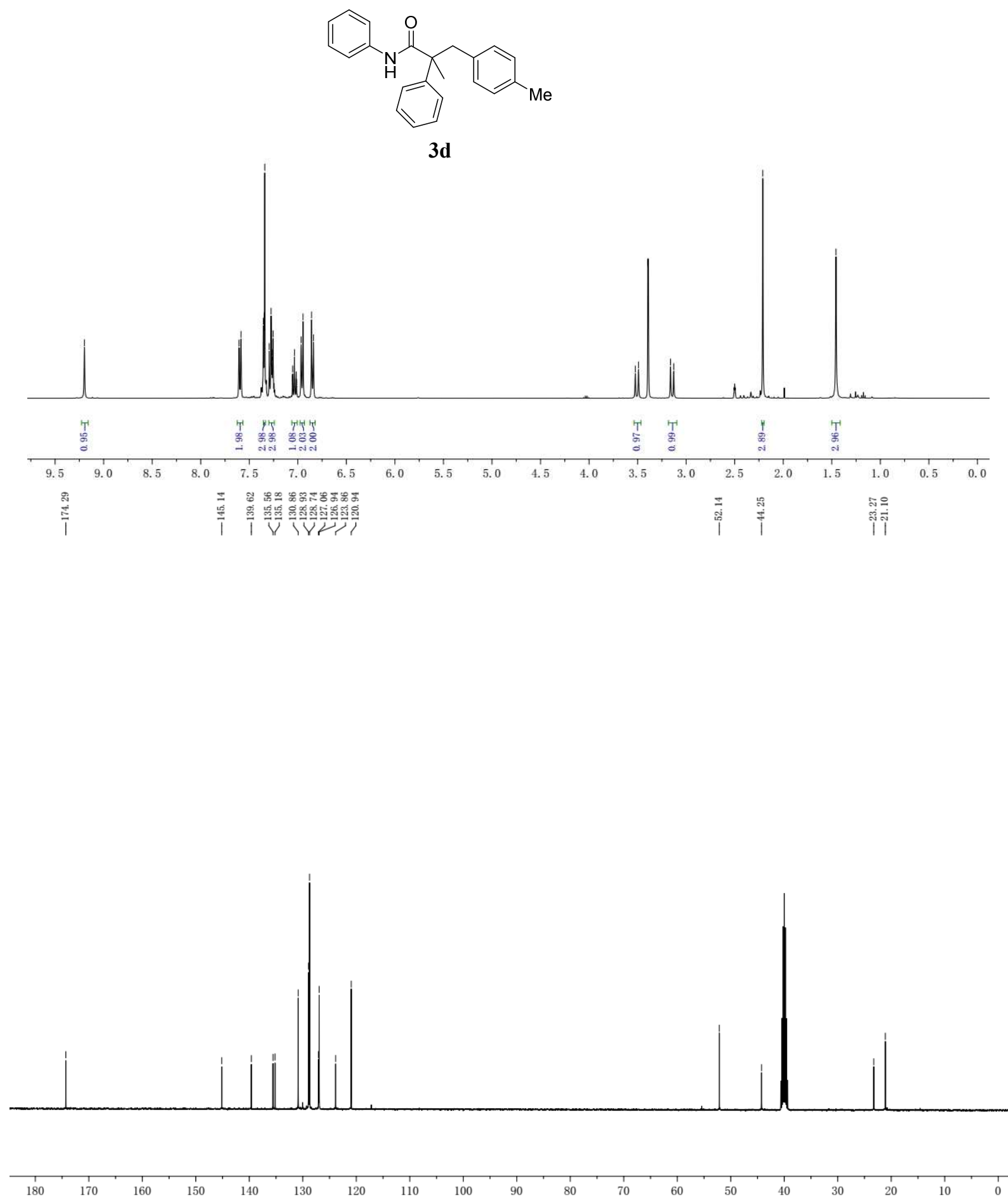
$\underset{\substack{0 \\ 0}}{\substack{0 \\ 0}}$

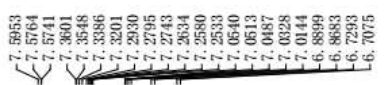

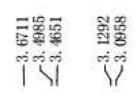

$\underset{1}{+}$
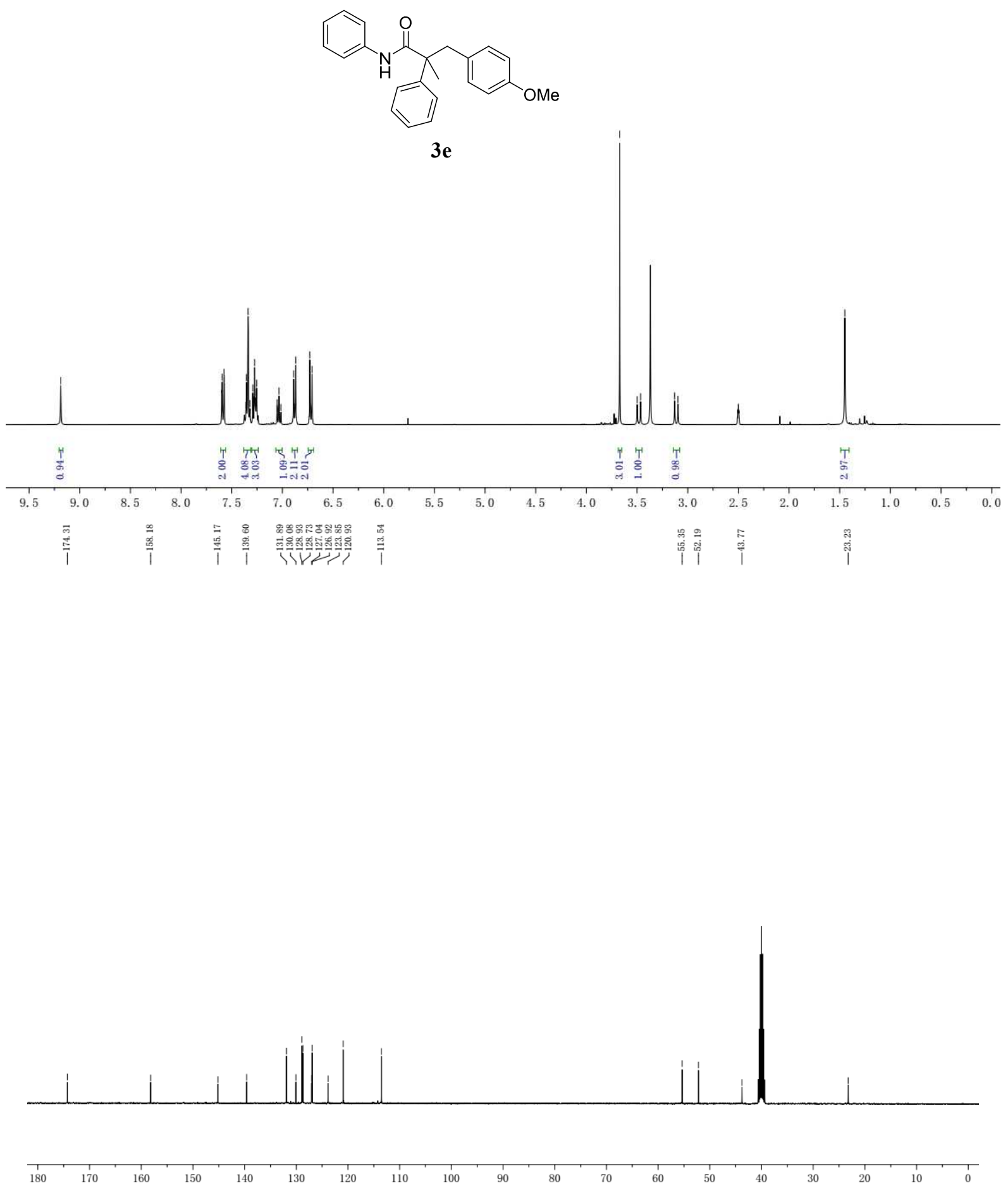

16 

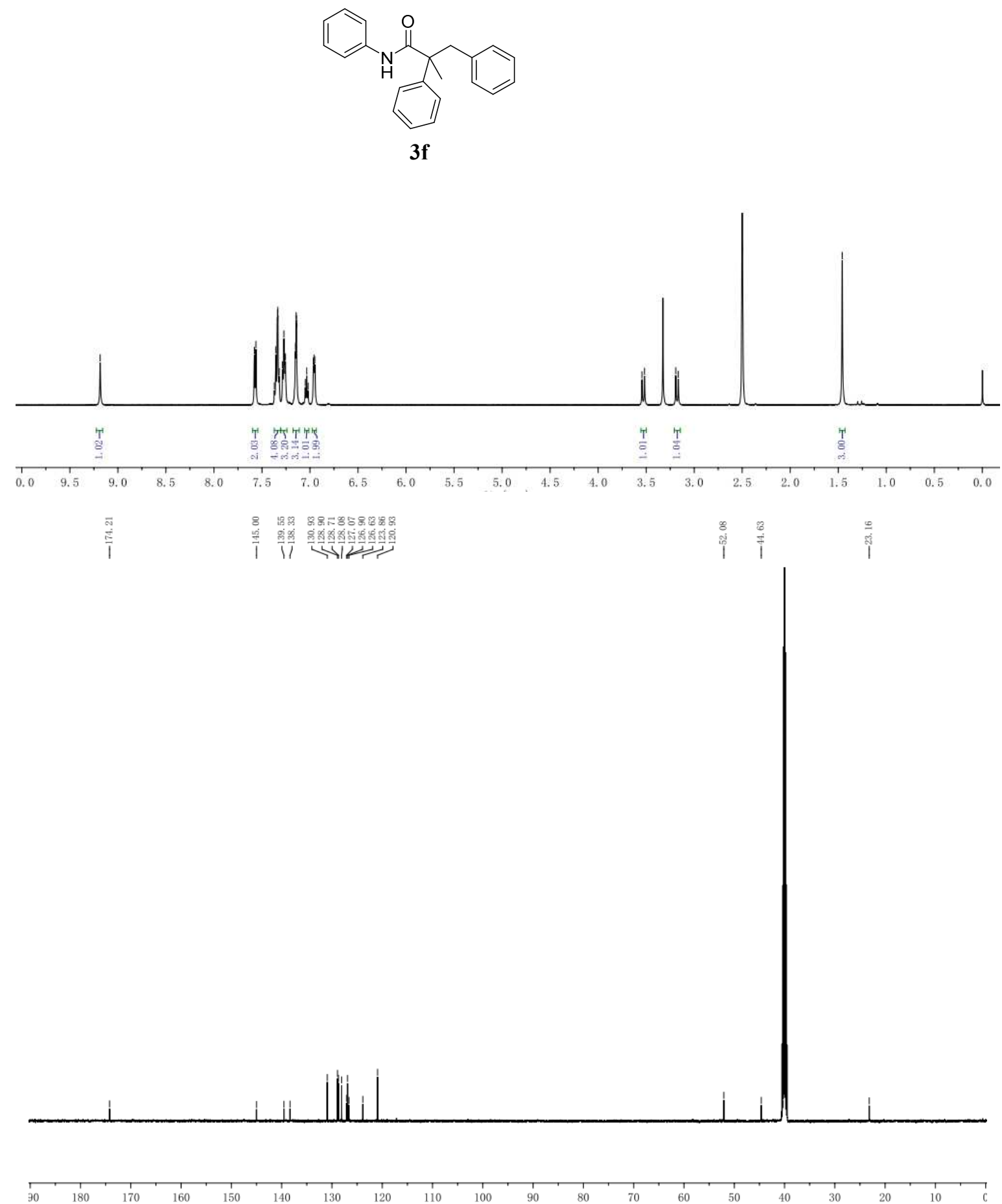

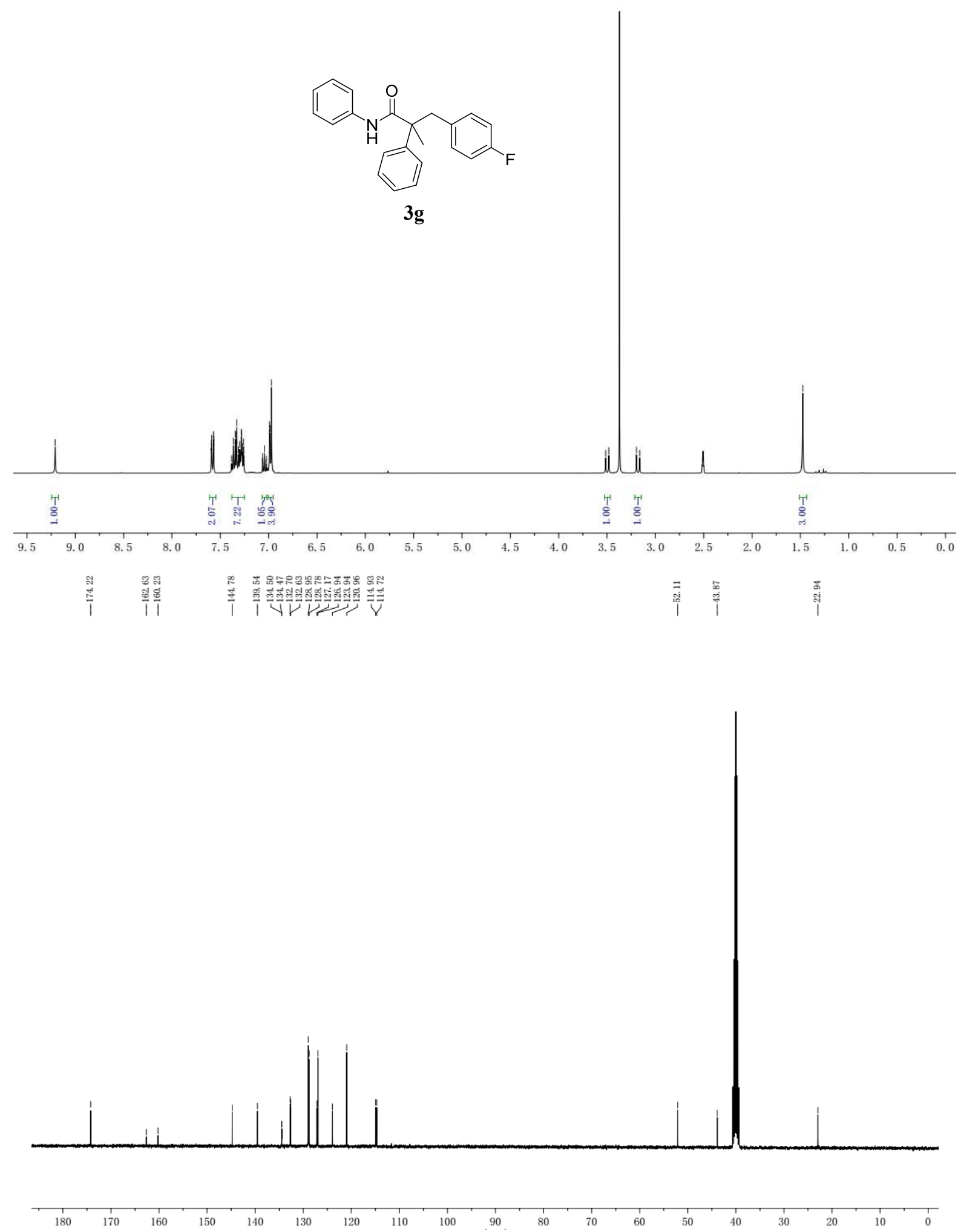


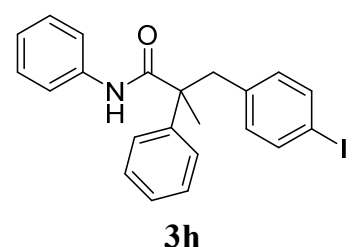

3h
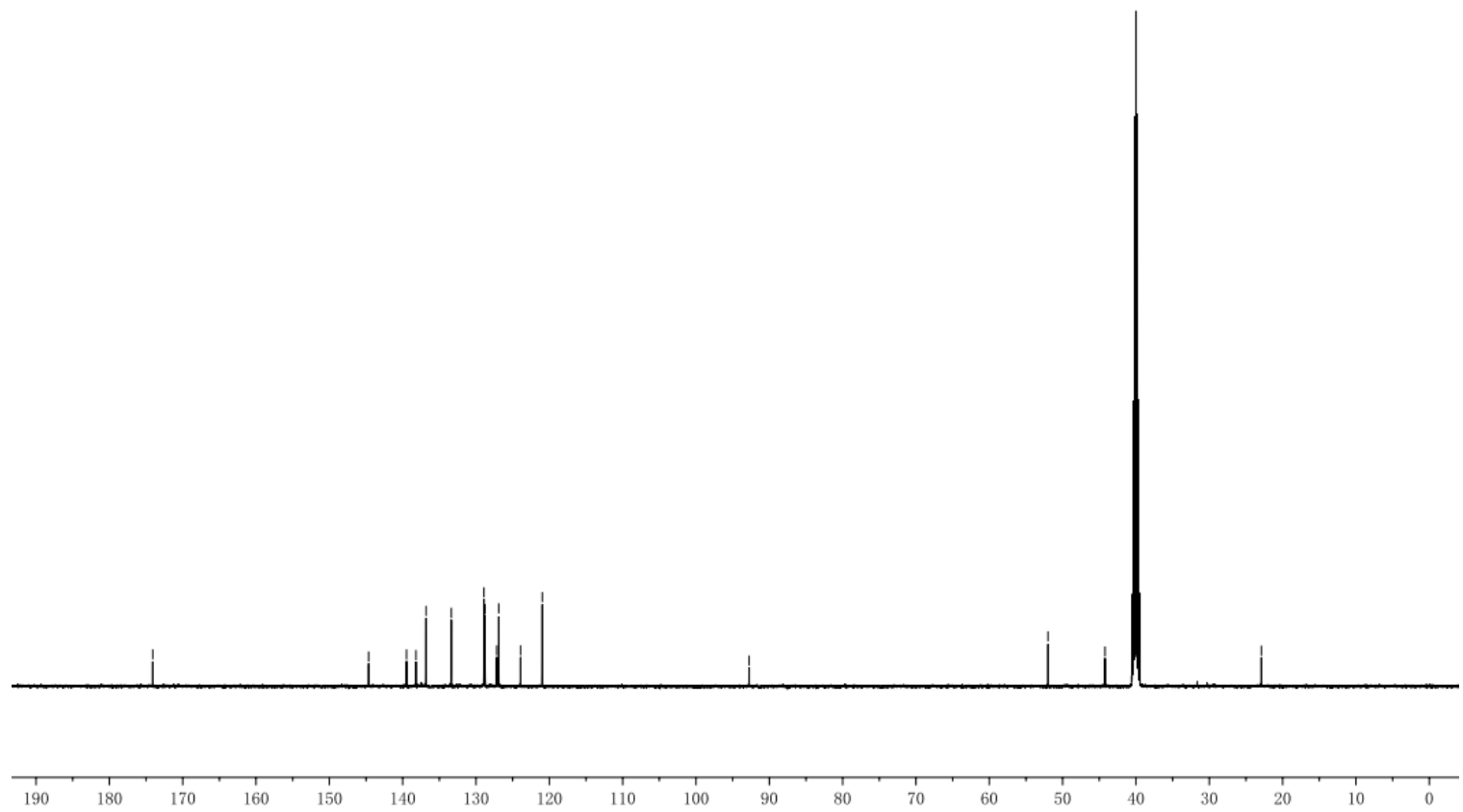
㐘

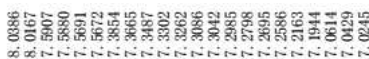

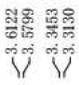

$\stackrel{\text { T. }}{1}$
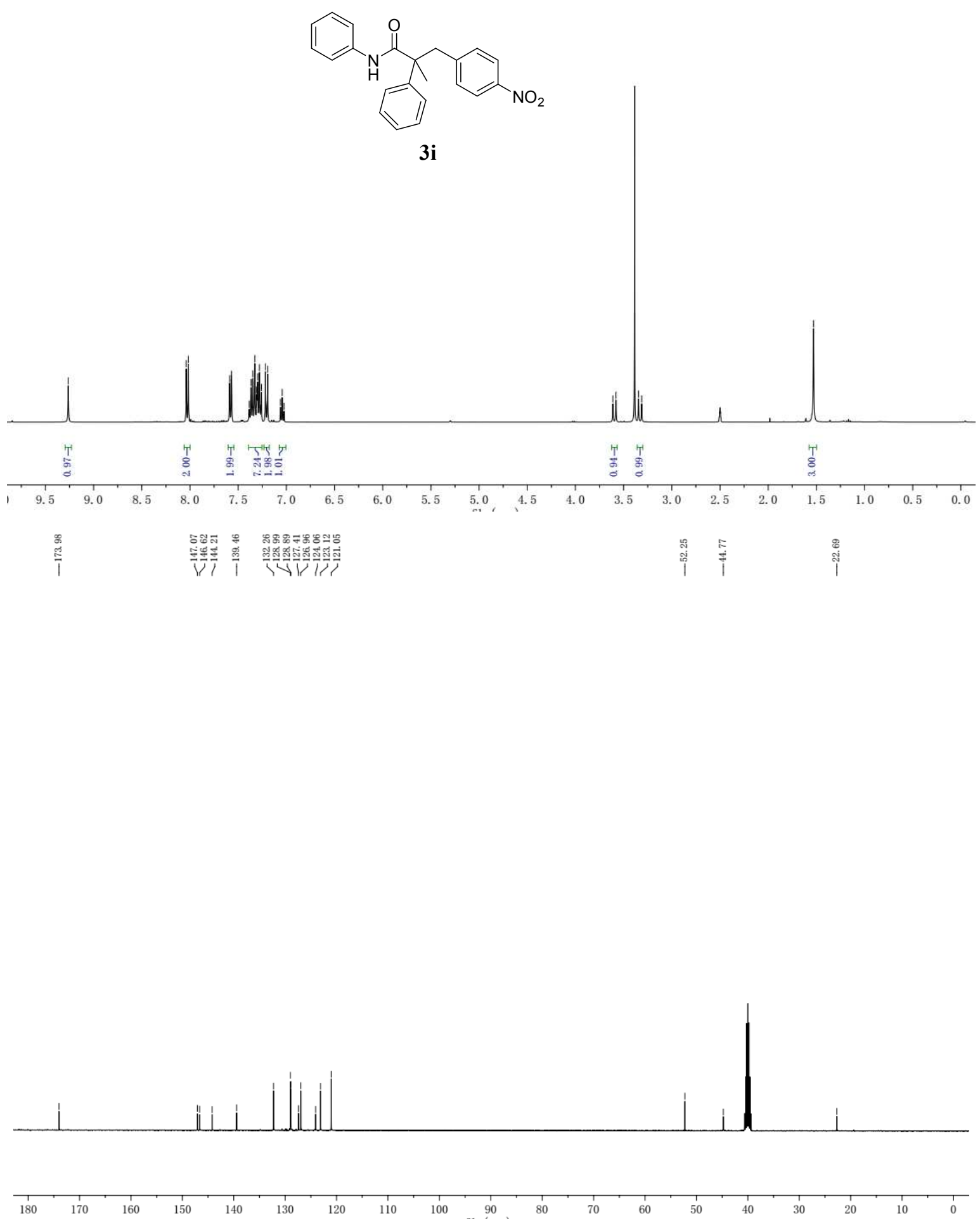

20 


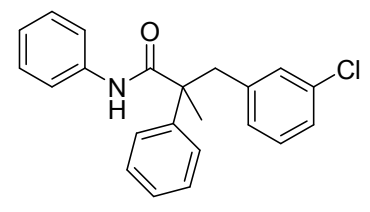

$3 \mathbf{j}$
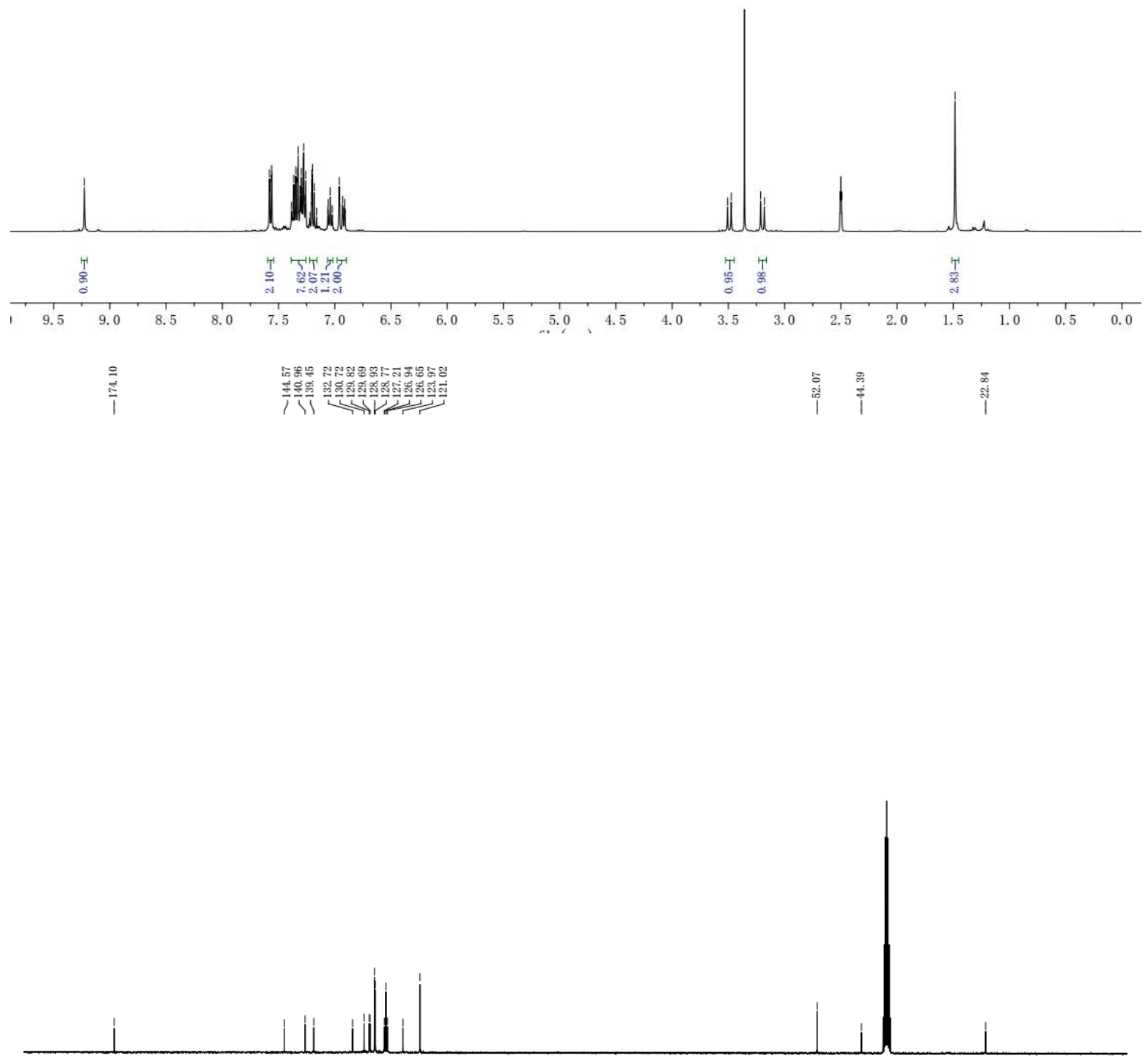

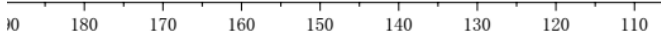




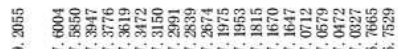

iा

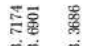

पंग
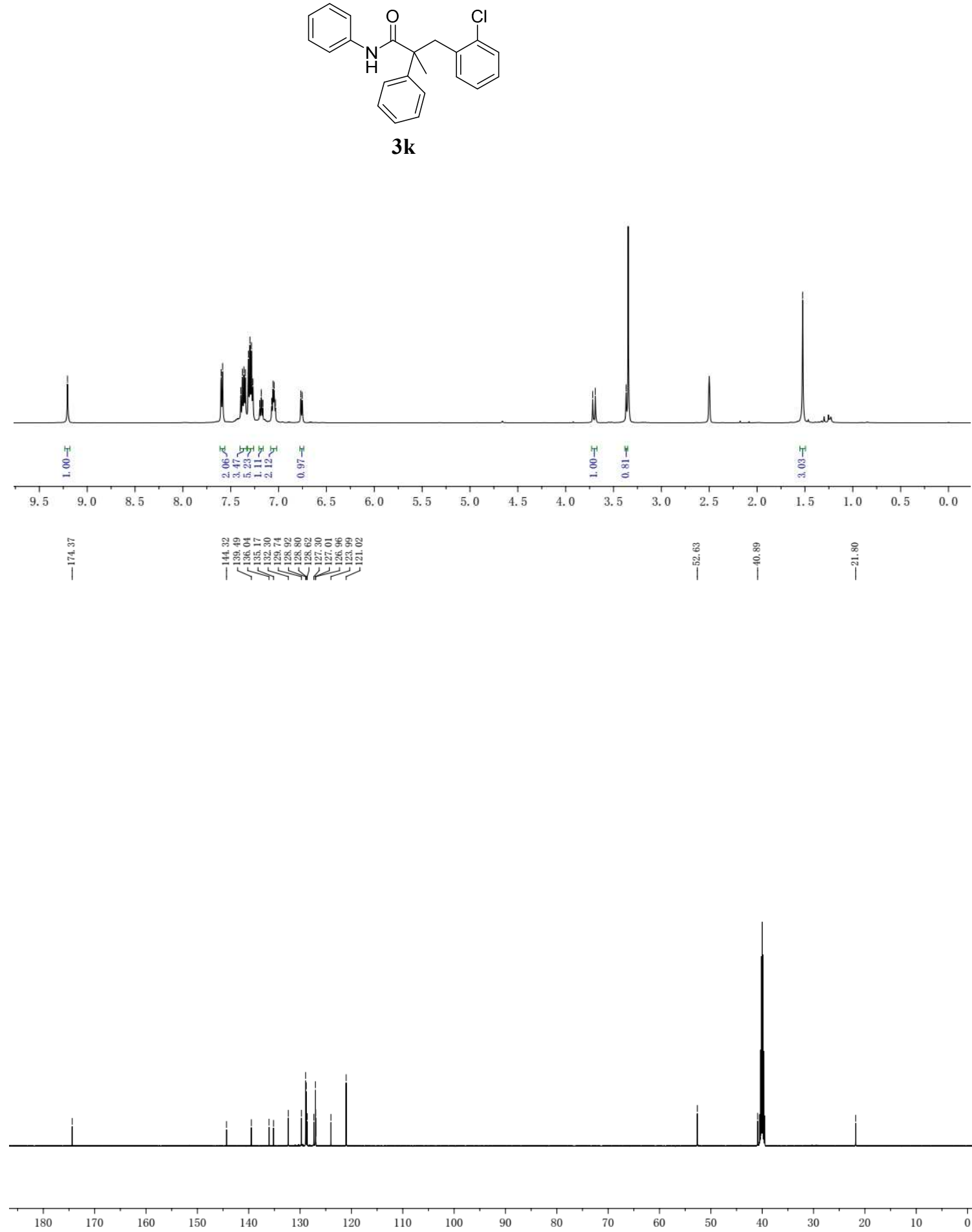


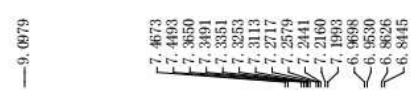

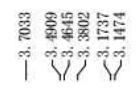

i

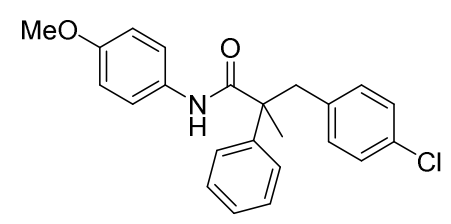

31
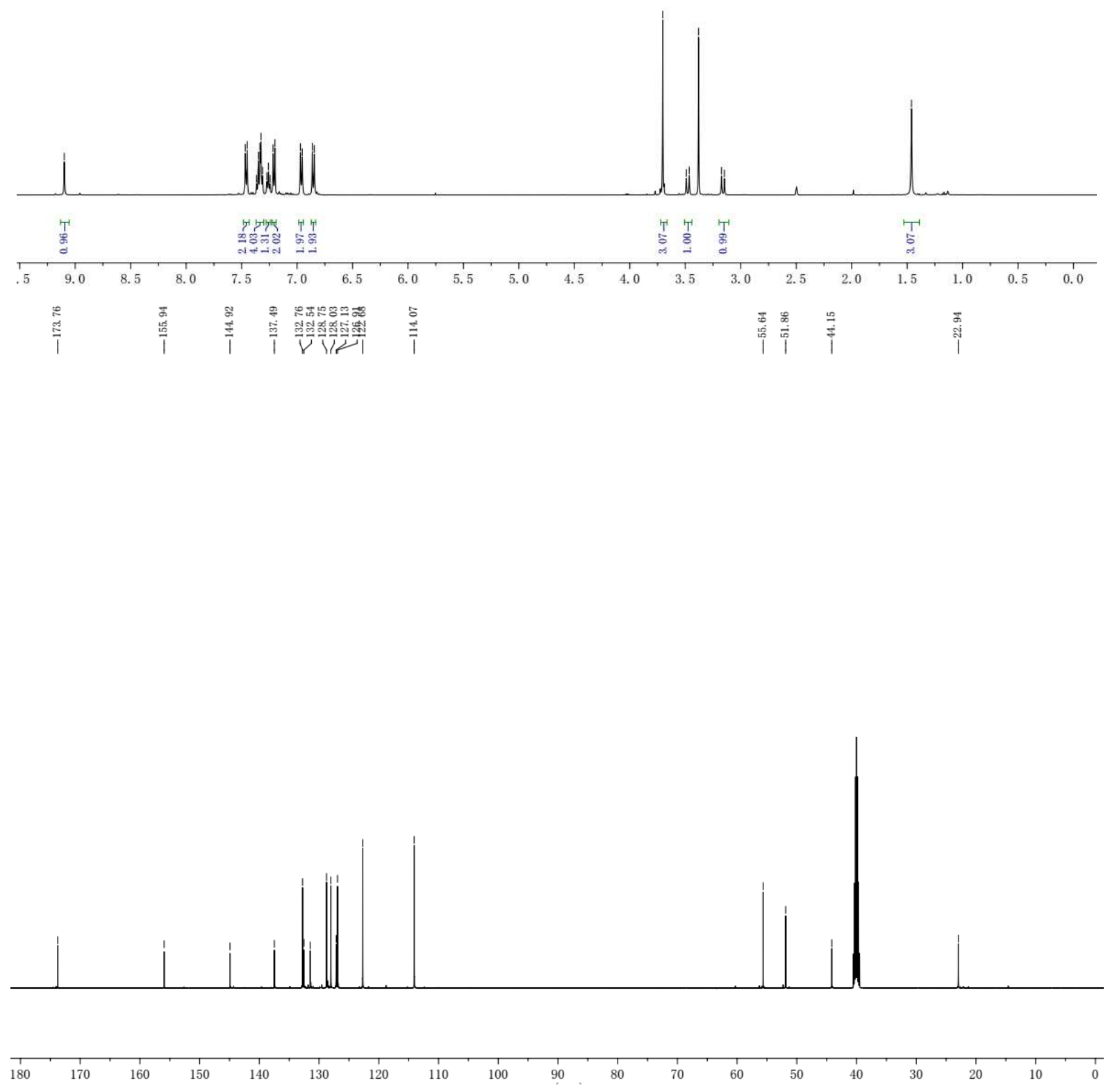

23 


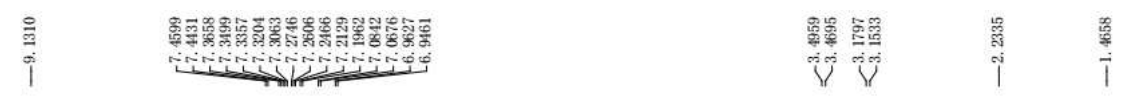

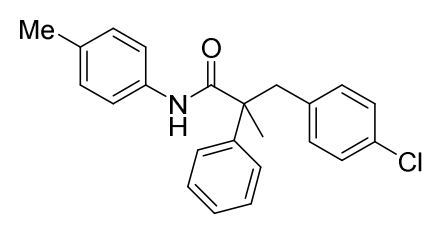

$3 \mathrm{~m}$
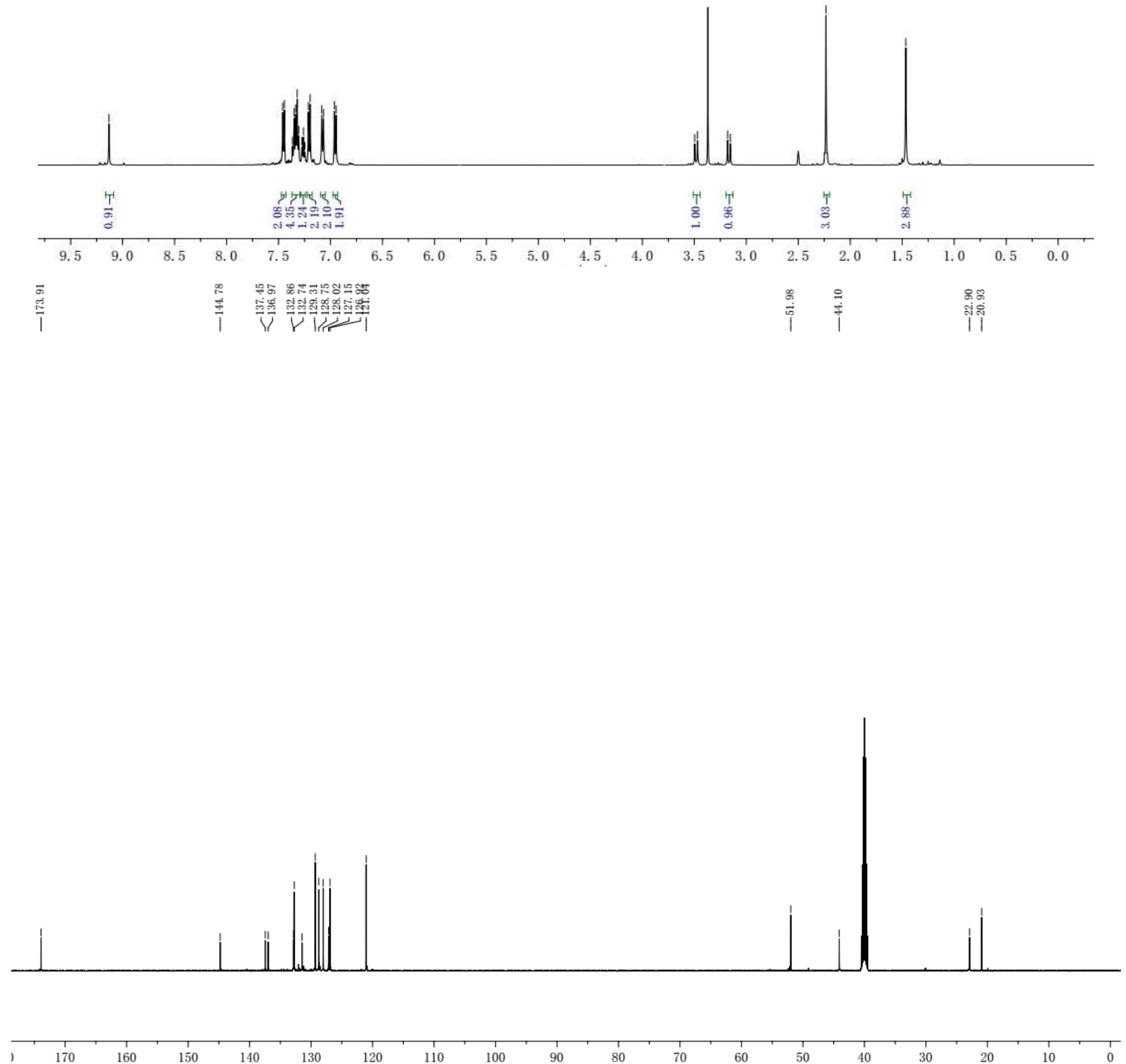


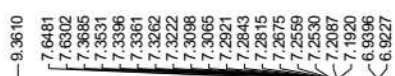

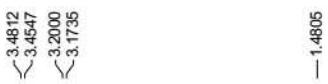

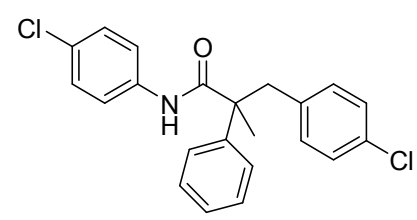

3n
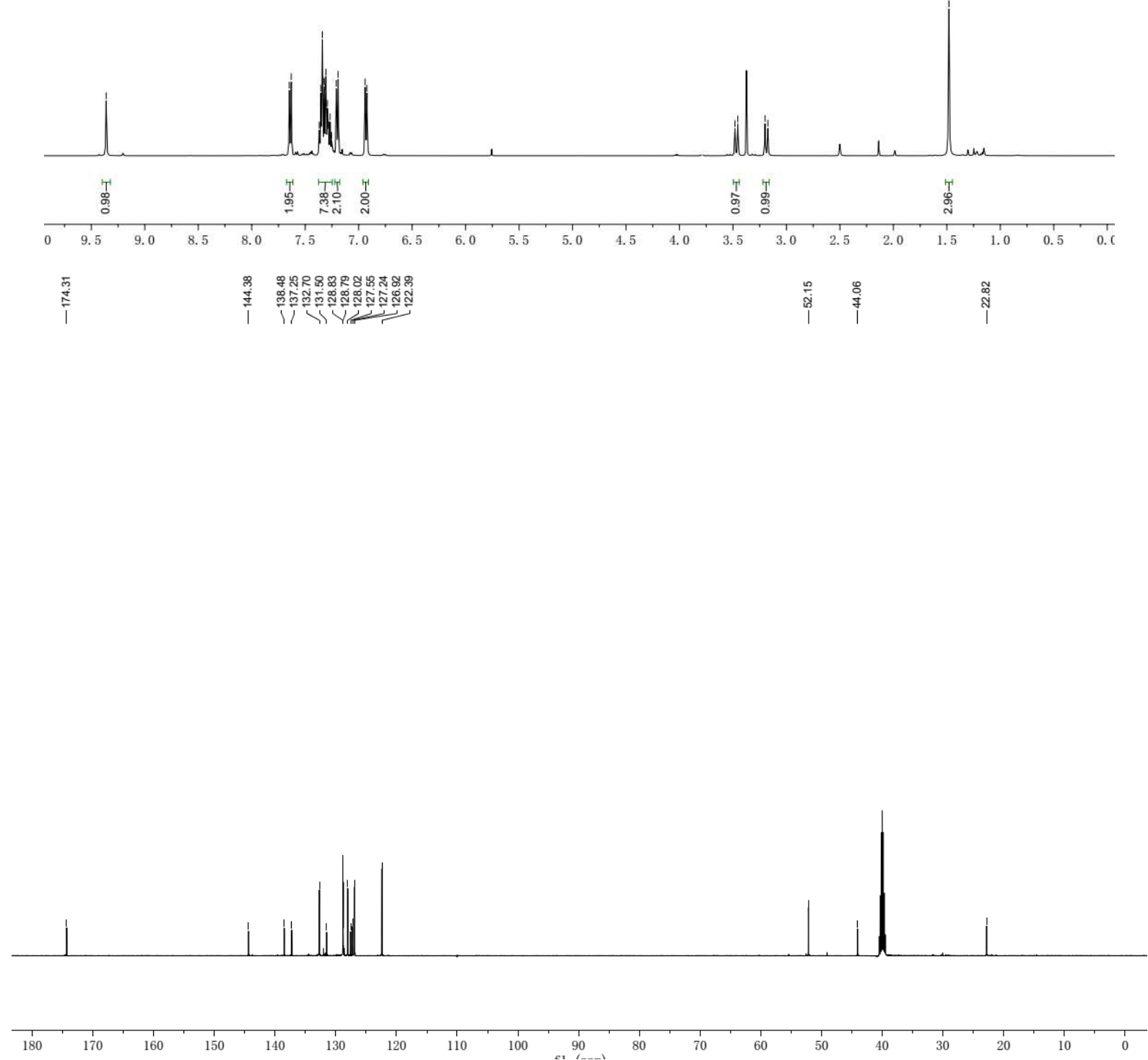


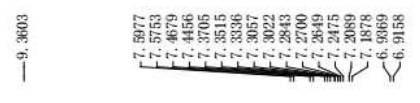

可送墨题

पif

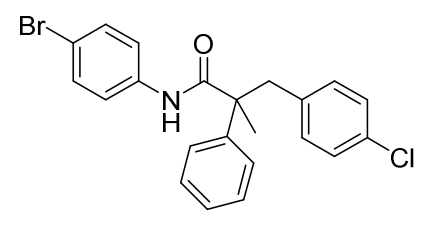

30
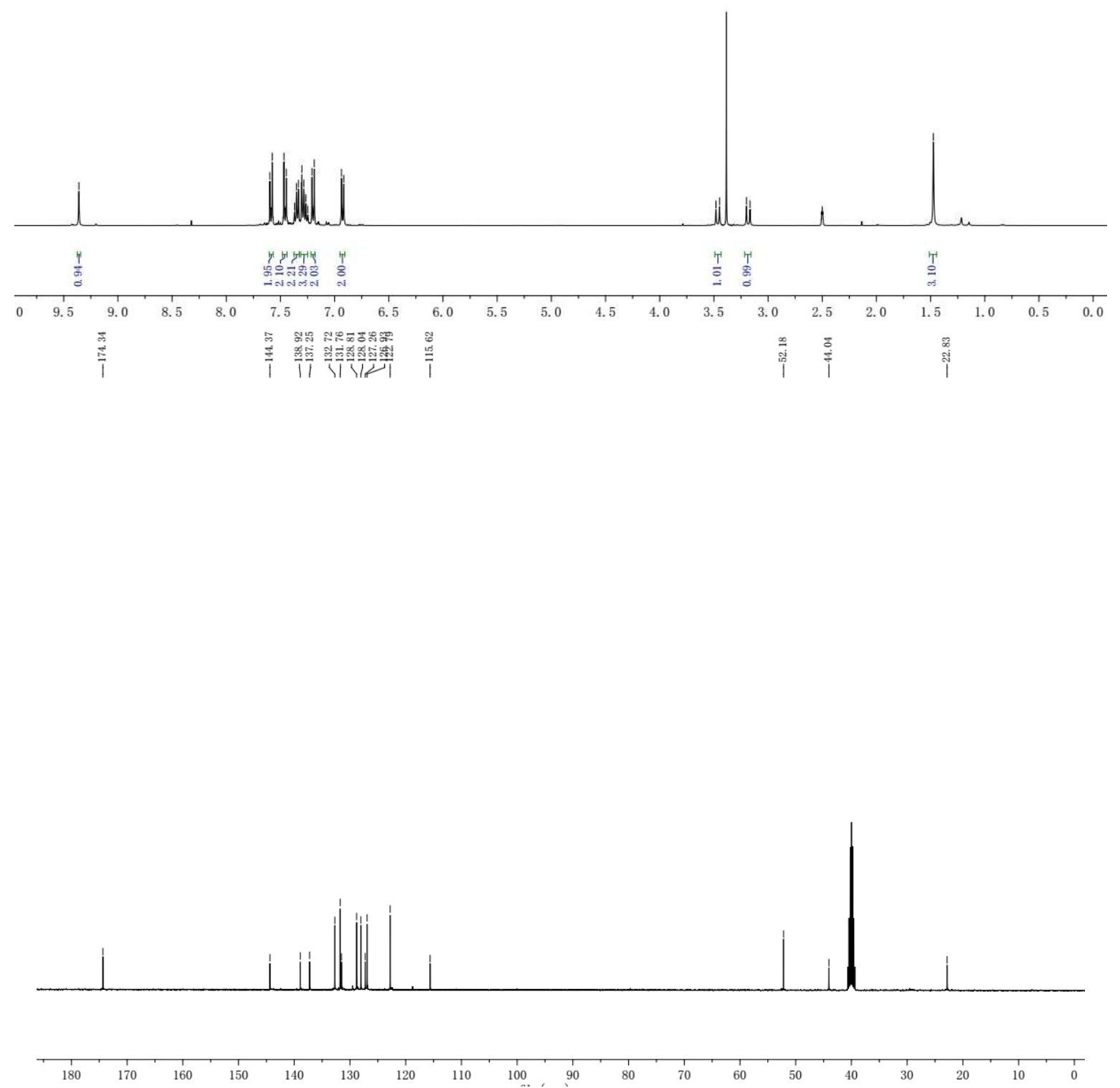


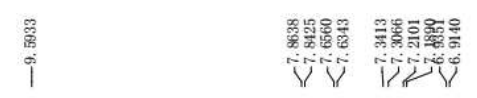

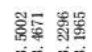

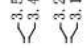

$\stackrel{5}{+}$

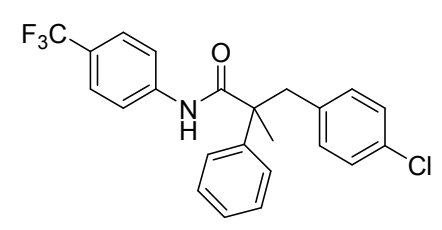

$3 p$
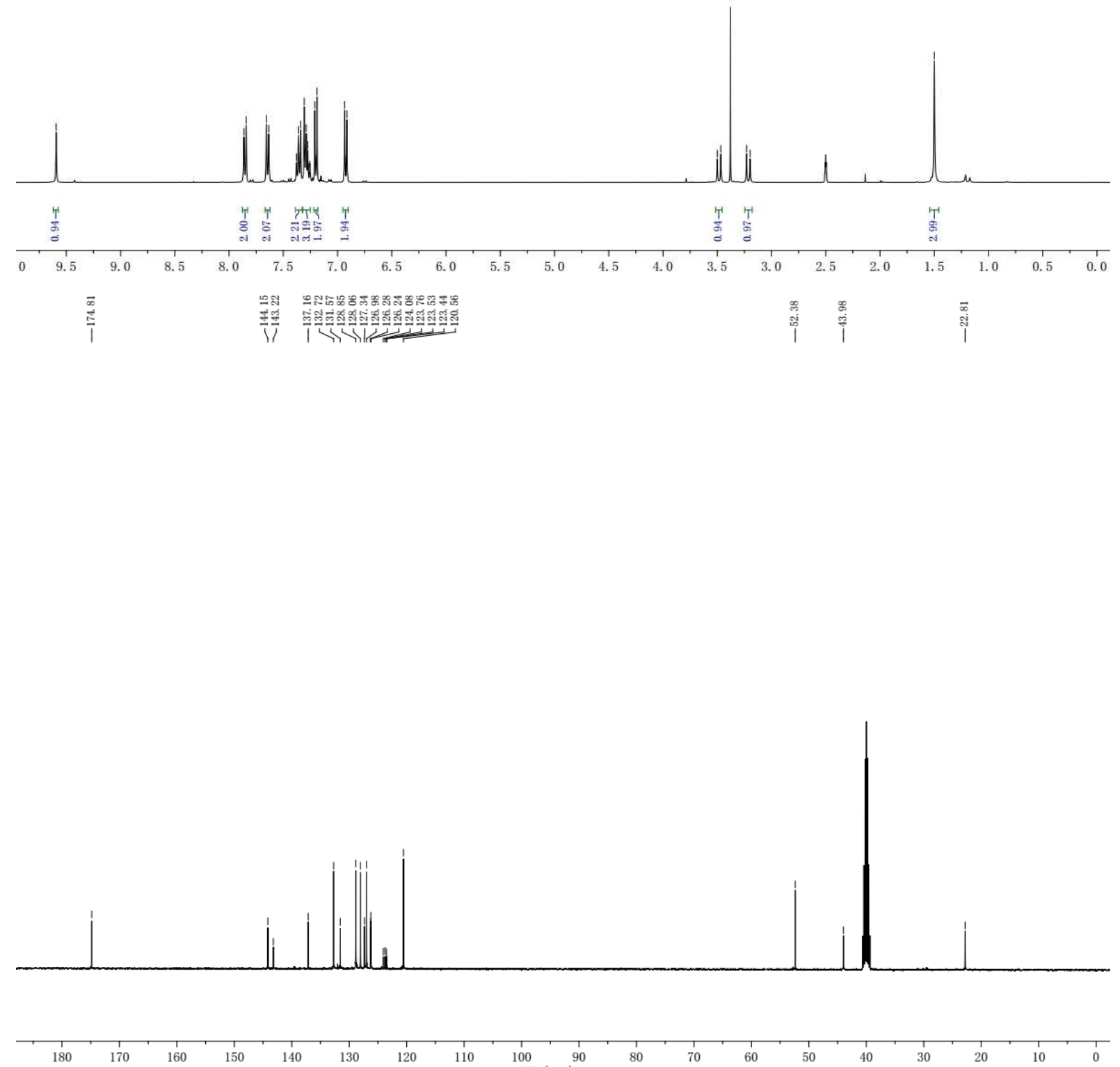

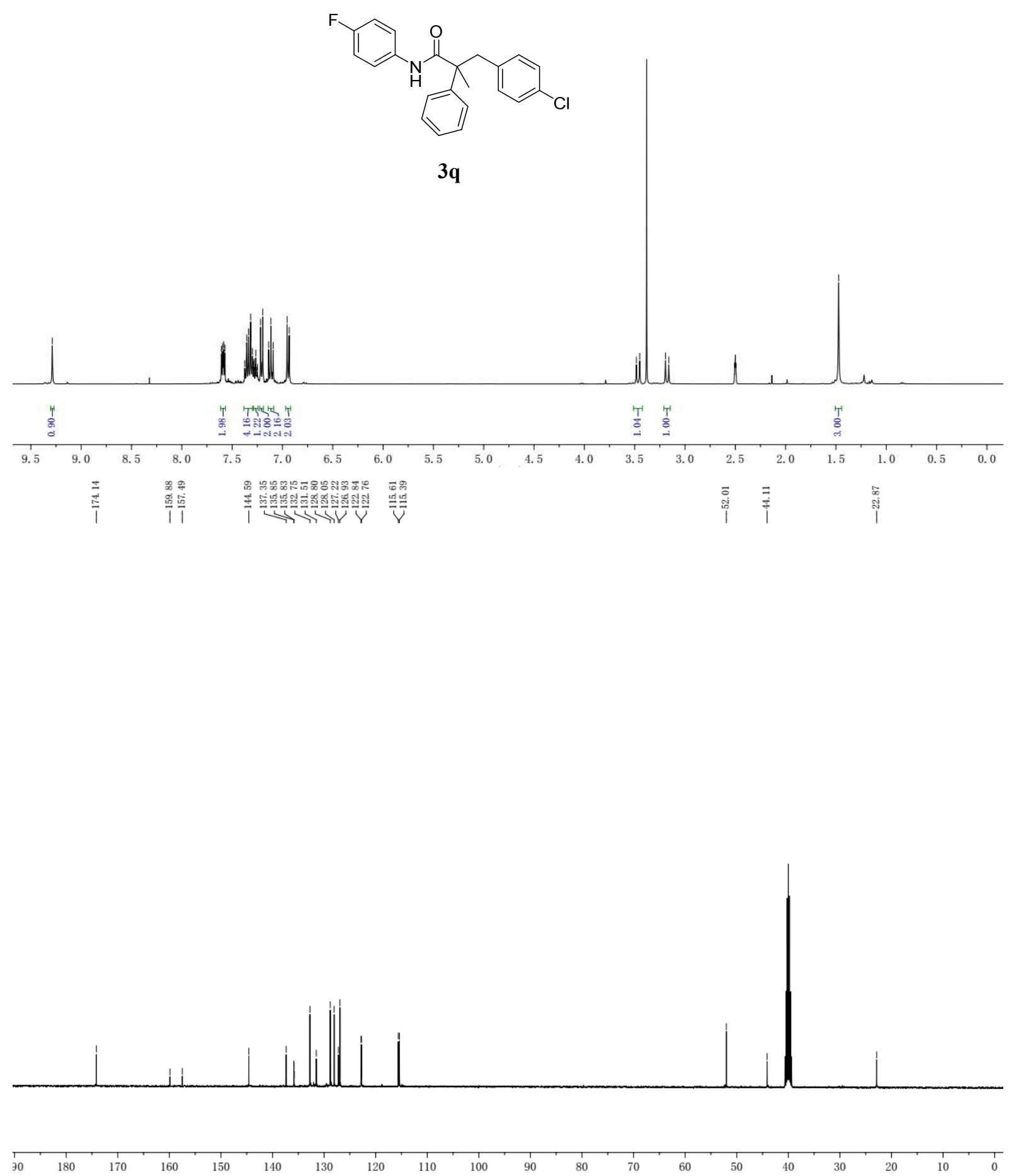


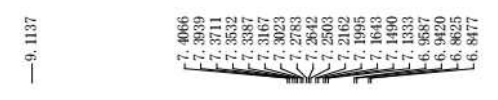

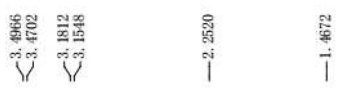
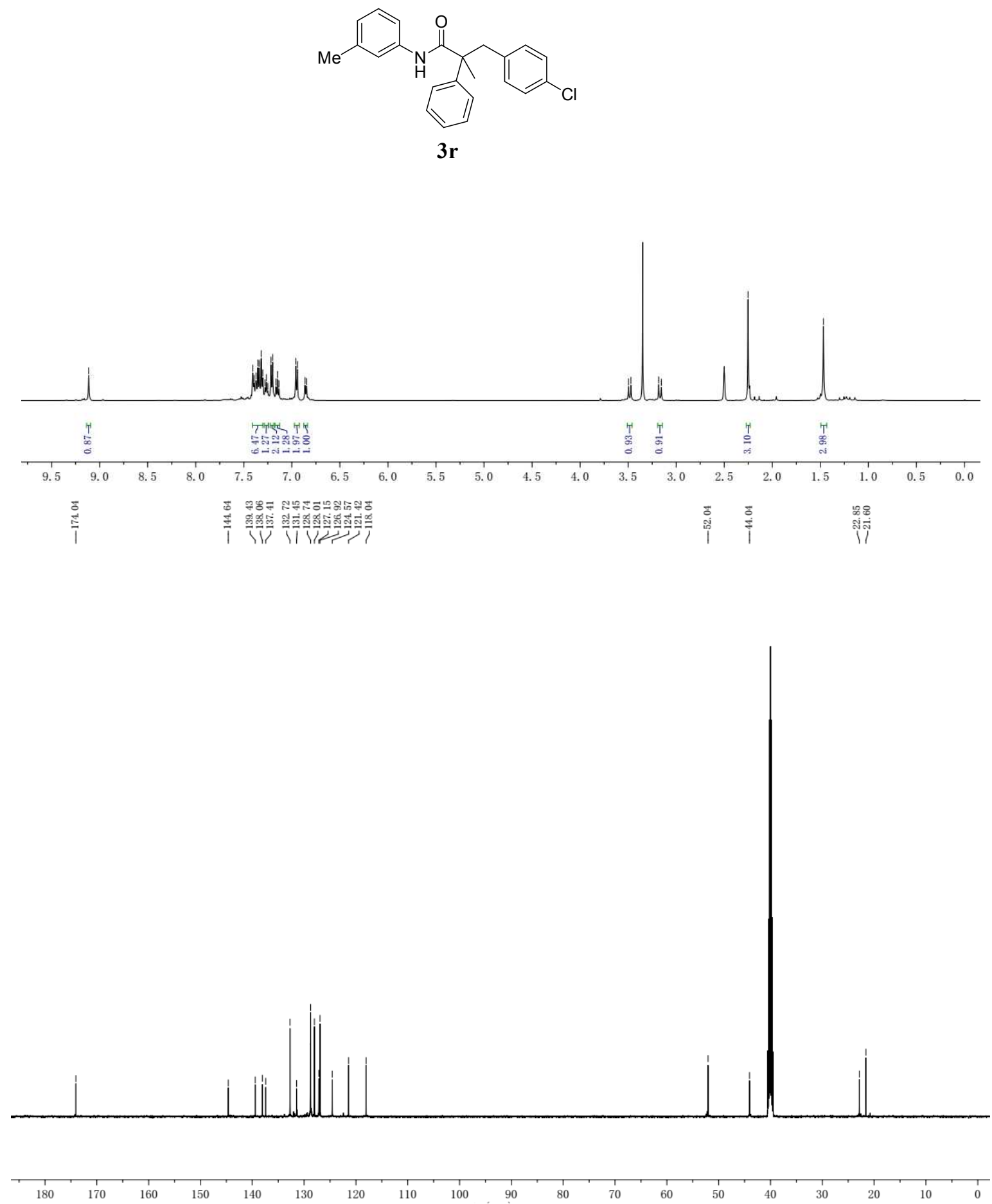


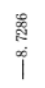

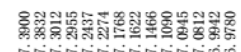

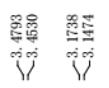

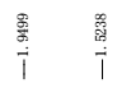
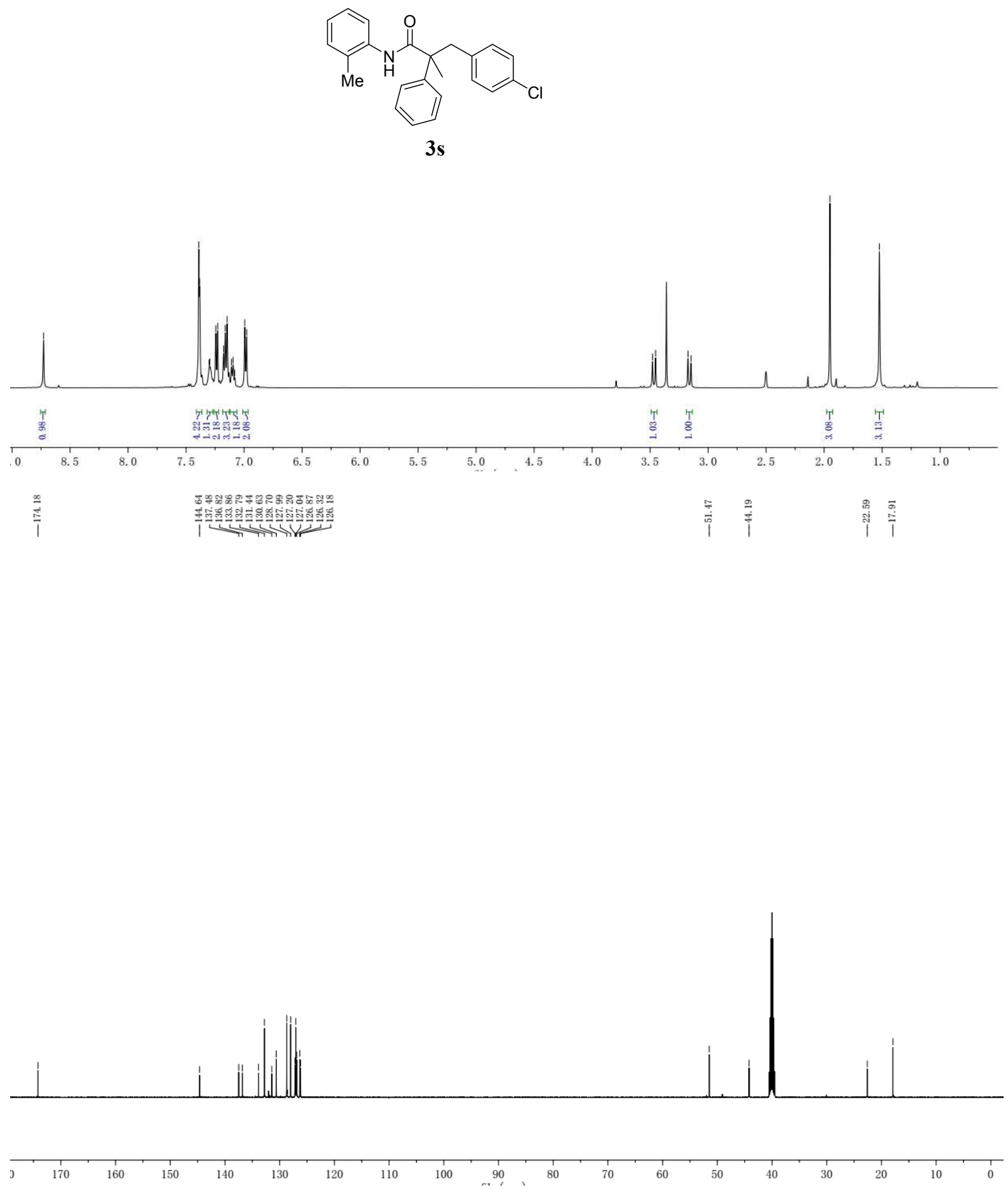

30 

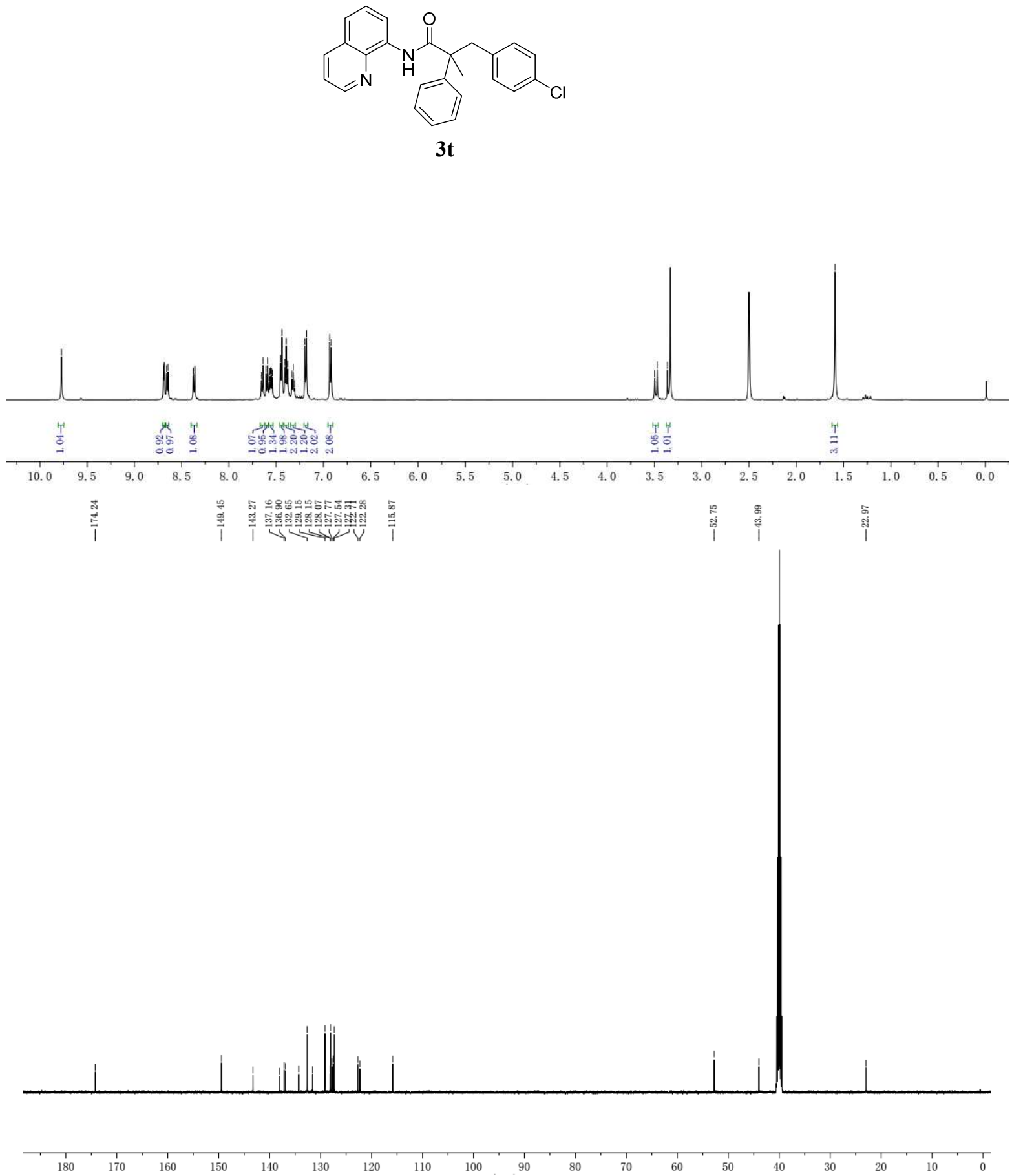
$\frac{8}{9}$

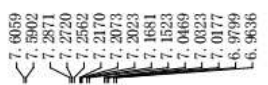

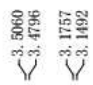

i
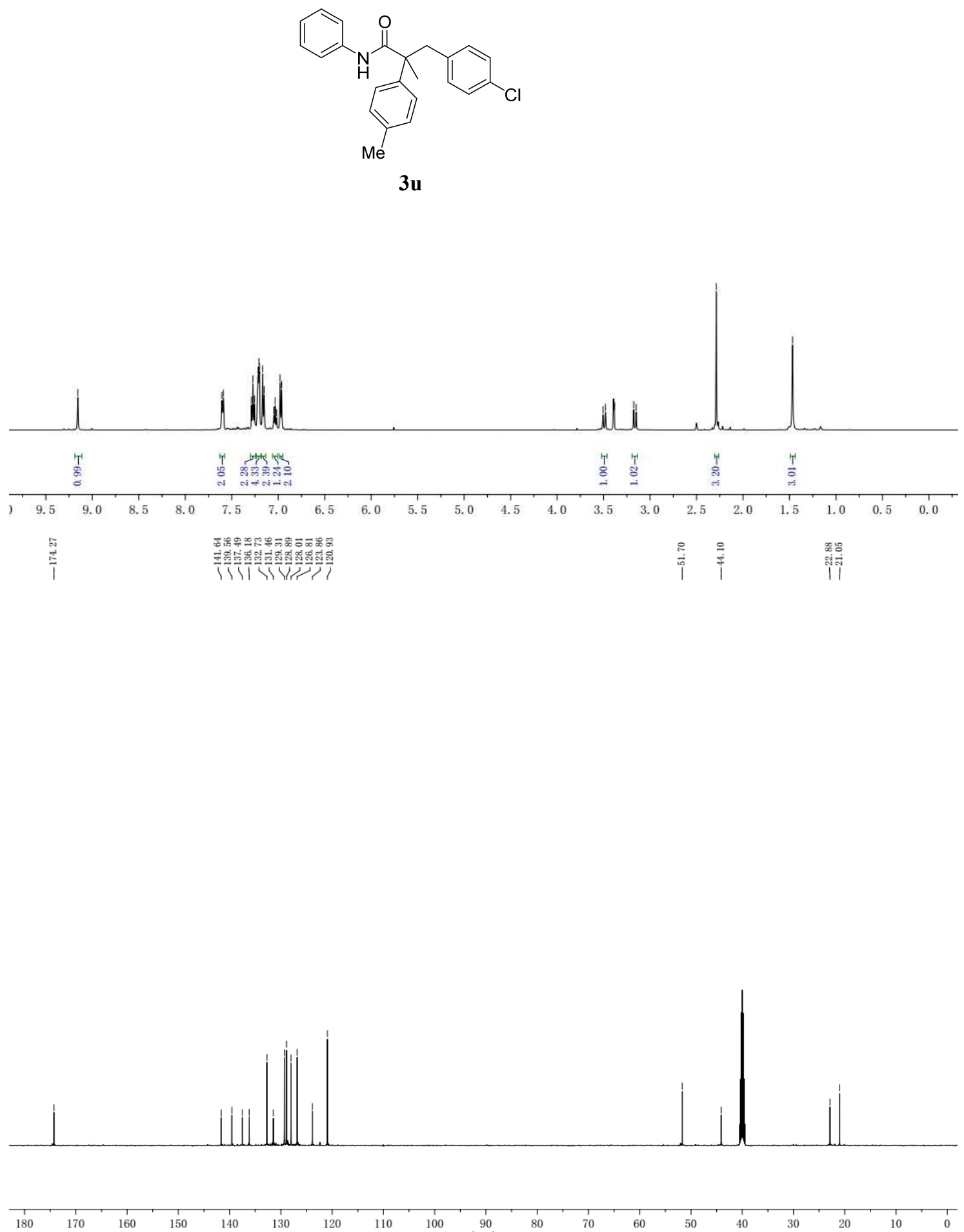

32 


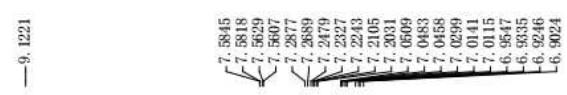

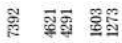

of ซึ
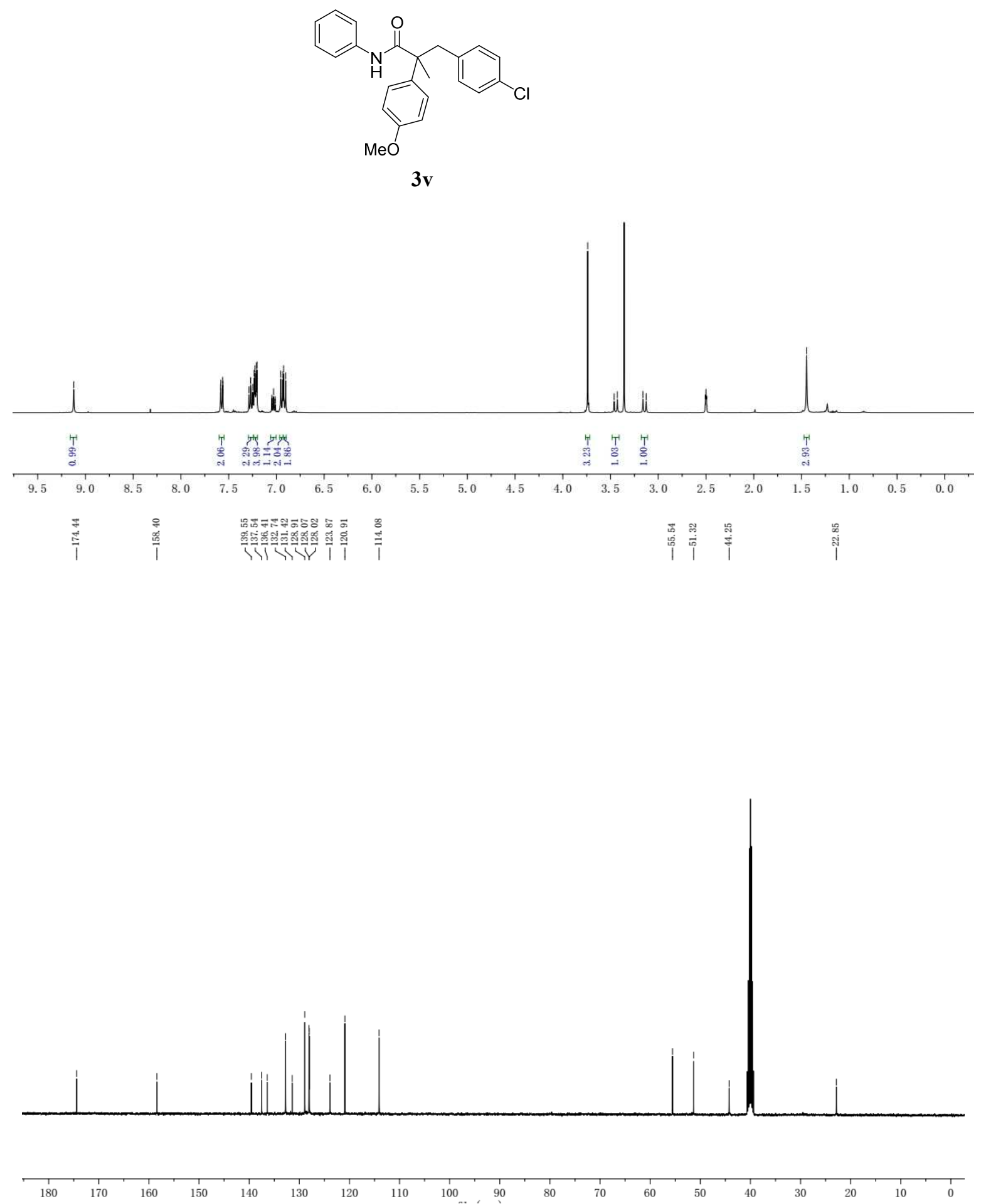


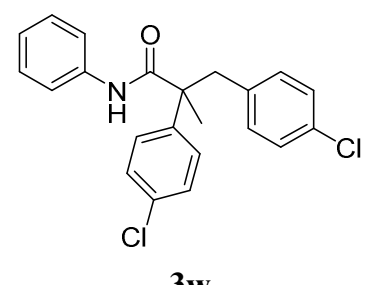

3w
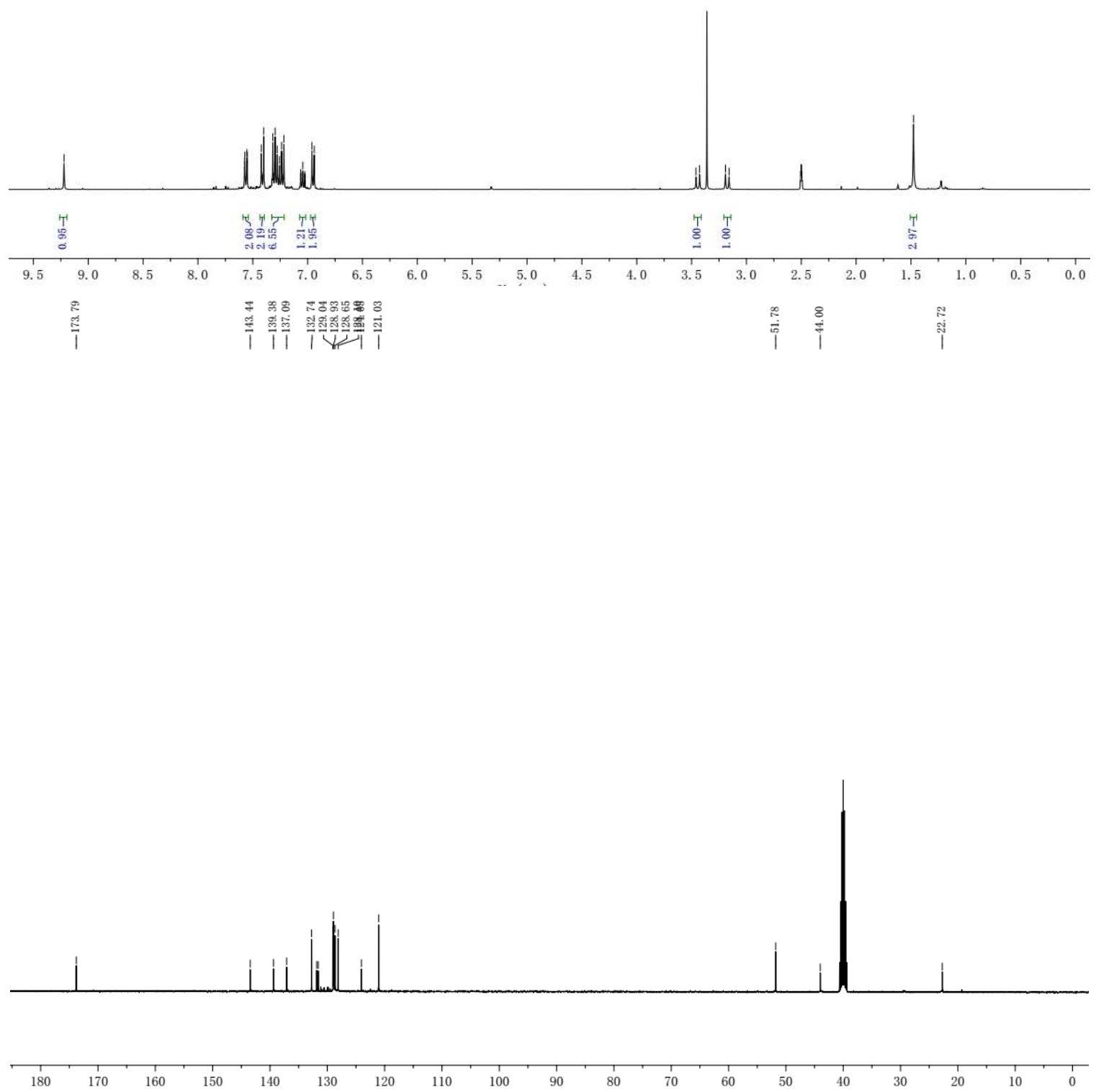
菏

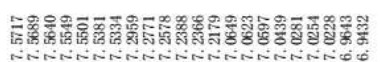

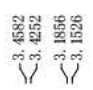

箞
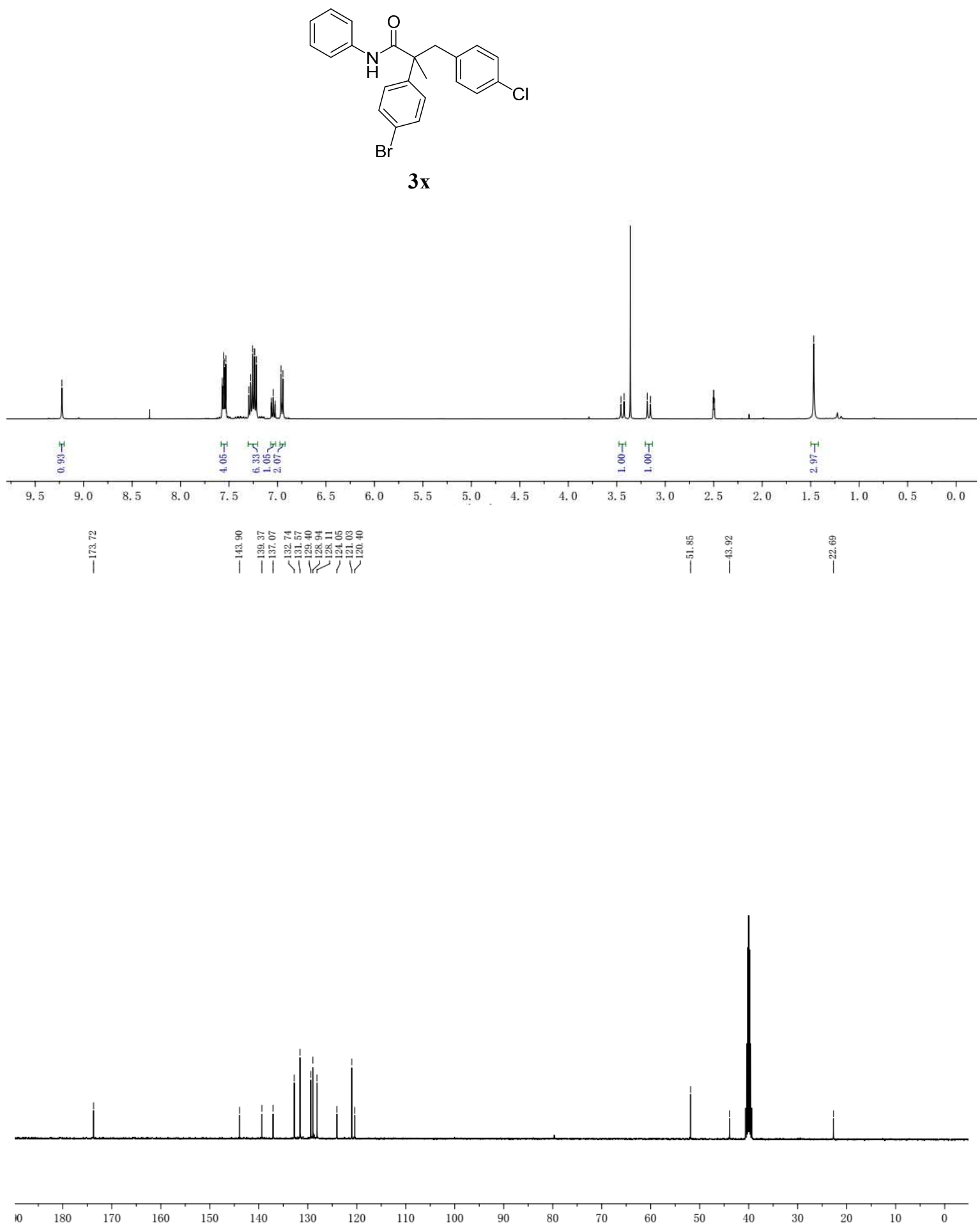

35 

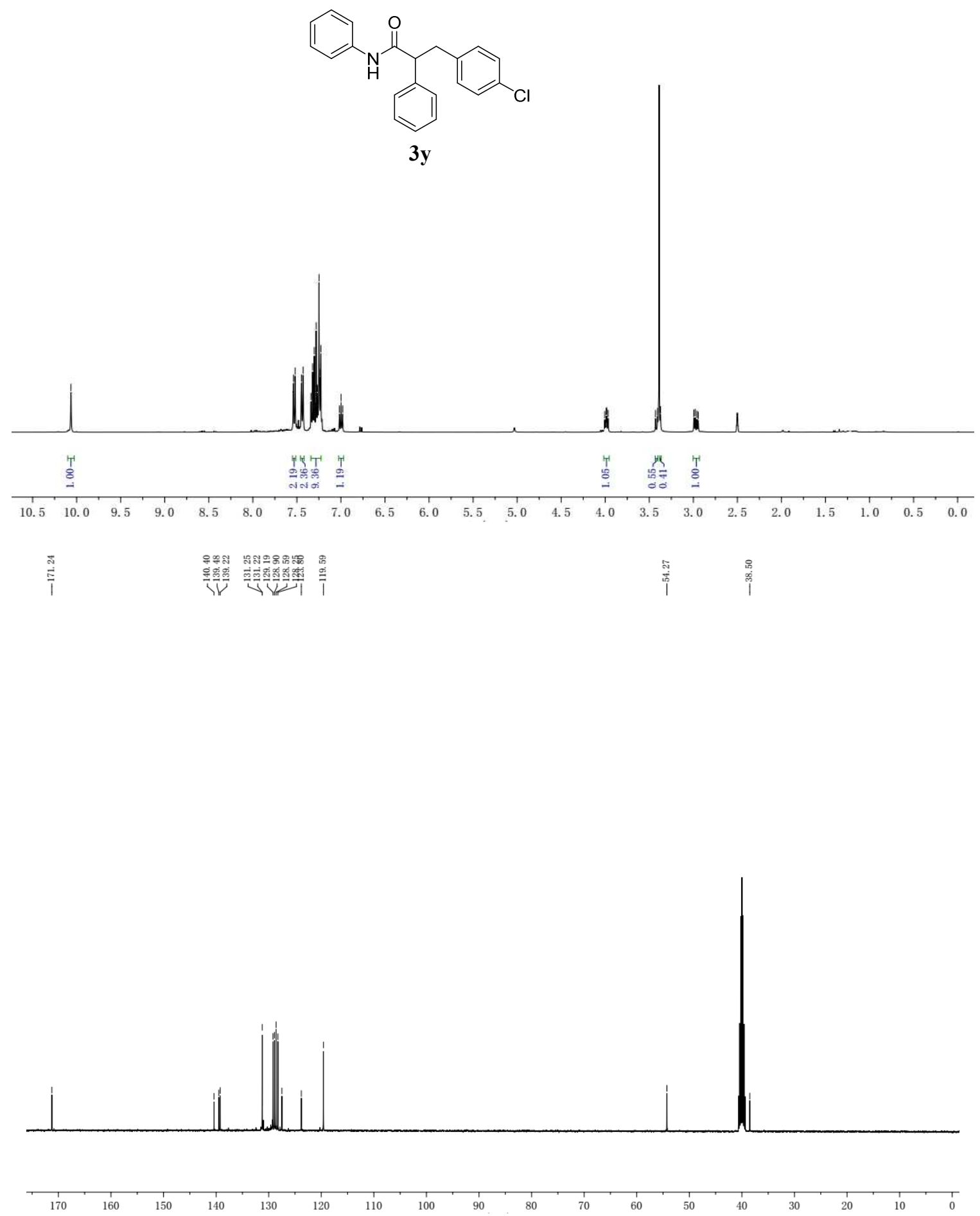